\title{
Dietary and Lifestyle Factors Related to Gastroesophageal Reflux Disease: A Systematic Review
}

This article was published in the following Dove Press journal:

Therapeutics and Clinical Risk Management

\author{
Mei Zhang' \\ Zheng-Kun Hou ${ }^{2}$ \\ Zhi-Bang Huang' \\ Xin-Lin Chen (1D ${ }^{3}$ \\ Feng-Bin $\mathrm{Liu}^{2,4}$ \\ 'Graduate College, Guangzhou \\ University of Chinese Medicine, \\ Guangzhou, Guangdong, China; \\ ${ }^{2}$ Gastroenterology Department, The \\ First Affiliated Hospital of Guangzhou \\ University of Chinese Medicine, \\ Guangzhou, Guangdong, China; \\ ${ }^{3}$ Department of Preventive Medicine and \\ Health Statistics, College of Basic Medical \\ Science, Guangzhou University of \\ Chinese Medicine, Guangzhou, \\ Guangdong, China; ${ }^{4}$ Baiyun Hospital of \\ the First Affiliated Hospital of Guangzhou \\ University of Chinese Medicine, \\ Guangzhou, Guangdong, China
}

Correspondence: Zheng-Kun Hou Department of Gastroenterology, The First Affiliated Hospital, Guangzhou University of Chinese Medicine,

Guangzhou, 510405, Guangdong, China Tel +86 020-3659/3।4

Email fenghou5I28@I26.com

\begin{abstract}
We performed this review to clarify which dietary and lifestyle factors are related to gastroesophageal reflux disease. Through a systematic search of the PubMed, EMBASE, China National Knowledge Infrastructure (CNKI), and Chinese BioMedical Literature (CBM) databases, we identified articles with clear definitions of GERD, including nonerosive gastroesophageal reflux disease (NERD), reflux esophagitis (RE) and Barrett's esophagus (BE), that included dietary and lifestyle factors as independent factors affecting the onset of GERD (expressed as odds ratios (ORs) or relative risks (RRs) and 95\% confidence intervals (CIs)). Due to heterogeneity among the studies, we used descriptive statistical analyses to analyze and synthesize each outcome based on the disease type. In total, 72 articles were included, conducted in ten Western countries (26 articles in total) and nine Eastern countries (46 articles in total). We categorized dietary factors into 20 items and lifestyle factors into 11 items. GERD is related to many irregular dietary and lifestyle habits (such as a habit of midnight snacking: $\mathrm{OR}=5.08,95 \% \mathrm{CI} 4.03-6.4$; skipping breakfast: $\mathrm{OR}=2.7,95 \% \mathrm{CI} 2.17-3.35$; eating quickly: $\mathrm{OR}=4.06,95 \% \mathrm{CI} 3.11-5.29$; eating very hot foods: $\mathrm{OR}=1.81,95 \% \mathrm{CI} 1.37-2.4$; and eating beyond fullness: $\mathrm{OR}=2.85,95 \%$ CI 2.18-3.73). Vegetarian diets (consumption of nonvegetarian food (no/yes); OR $=0.34,95 \% \mathrm{CI}$ $0.211-0.545)$ and no intake of meat $(\mathrm{OR}=0.841,95 \% \mathrm{CI} 0.715-0.990)$ were negatively related to GERD, while meat (daily meat, fish, and egg intake: $\mathrm{OR}=1.088,95 \%$ CI $1.042-1.135$ ) and fat (high-fat diet: $\mathrm{OR}=7.568,95 \% \mathrm{CI} 4.557-8.908$ ) consumption were positively related to GERD. An interval of less than three hours between dinner and bedtime (OR=7.45, 95\% CI 3.38-16.4) was positively related to GERD, and proper physical exercise (physical exercise $>30$ minutes $(>3$ times/week): $\mathrm{OR}=0.7,95 \%$ CI $0.6-0.9)$ was negatively correlated with GERD. Smoking $(\mathrm{OR}=1.19,95 \% \mathrm{CI} 1.12-1.264)$, alcohol consumption $(\mathrm{OR}=1.278,95 \%$ CI $1.207-1.353)$ and mental state (poor mental state: $\mathrm{OR}=1.278,95 \% \mathrm{CI} 1.207-1.353$ ) were positively correlated with GERD. RE (vitamin C: $\mathrm{OR}=0.46,95 \% \mathrm{CI}=0.24-0.90$ ) and $\mathrm{BE}$ (vitamin $\mathrm{C}$ : $\mathrm{OR}=0.44,95 \% \mathrm{CI}$ 0.2-0.98; vitamin $\mathrm{E}$ : $\mathrm{OR}=0.46,95 \% \mathrm{CI} 0.26-0.83$ ) were generally negatively correlated with antioxidant intake. In conclusion, many dietary and lifestyle factors affect the onset of GERD, and these factors differ among regions and disease types. These findings need to be further confirmed in subsequent studies.
\end{abstract}

Keywords: gastroesophageal reflux disease, diet, lifestyle, systematic review

\section{Introduction}

Gastroesophageal reflux disease (GERD) is a type of chronic gastrointestinal disease in which heartburn and regurgitation are the main clinical manifestations and esophageal and pulmonary symptoms may occur. According to endoscopic findings and esophageal $\mathrm{pH}$ monitoring, there may also be 
asymptomatic manifestations. ${ }^{1}$ The main pathological mechanism is the invasion of stomach contents and dysfunction of the esophageal antireflux barrier. The former is mainly caused by the formation of gastric acid pockets and delayed gastric emptying. The latter is mainly caused by dysfunction of the lower esophageal sphincter (LES). The frequency of transient lower esophageal sphincter relaxation (TLESR) increases, and esophageal acid removal dysfunction occurs, among other factors, but the specific causes of these factors are currently unclear. ${ }^{2}$ The incidence rate is increasing annually, but there are substantial differences among regions. The highest incidence, which occurs in Europe and the United States, reaches 20\%, and the lowest incidence, which occurs in Asia, is approximately $10 \%$. Additionally, age, sex, race, genes, and factors related to diet and lifestyle (such as obesity and smoking) are related to GERD., Effective treatments include lifestyle modification, proton pump inhibitors (PPIs) and surgery. With the recognition of the side effects of the long-term use of PPIs, as nondrug methods and first-line treatments, diet and lifestyle modifications are receiving increasing attention due to their importance in the prevention and treatment of GERD..$^{5-7}$

Therefore, we reviewed and analyzed the current literature to elucidate which dietary and lifestyle factors are associated with GERD, provide specific and informative dietary and lifestyle recommendations for patients, and provide information for follow-up studies. We also attempt to elucidate the dietary and lifestyle differences associated with GERD between Western countries and Eastern countries.

\section{Materials and Methods}

\section{Search Strategy}

We conducted a comprehensive literature search in two English databases, ie, PubMed and EMBASE, and two Chinese databases, ie, the Chinese BioMedical Literature (CBM) and China National Knowledge Infrastructure (CNKI) databases, with appropriate search strategies. In PubMed, the search scope was limited to titles and abstracts, and we searched for articles related to diet and lifestyle using words such as diet, food, lifestyle, and nutrition. Free words and $\mathrm{MeSH}$ terms were used to perform the searches, and the search terms were connected by "and". Searches of
English-language databases were limited to articles published in English articles that involved humans. We searched for articles published prior to 2020-03-01. The Chinese databases were searched by journal, and the search was limited to the core journals. We searched for articles published before 2020-03-14. The details of the search strategy for each database are shown in Appendix 1.

\section{Data Selection}

The inclusion criteria were as follows: (1) clear definition of GERD, nonerosive gastroesophageal reflux disease (NERD), reflux esophagitis (RE) or Barrett's esophagus (BE) and (2) articles involving diet and lifestyle as independent factors affecting the onset of GERD. Articles that met any of the following criteria were excluded: (1) research subjects aged $<18$ years; (2) no full text available; (3) reviews, systematic reviews, pooled analyses, and meta-analyses; (4) articles focusing on GERD symptom relief, recurrence, progression, and complications; (5) articles that did not provide the odds ratio (OR) or hazard ratio (HR) and $95 \%$ confidence interval $(95 \% \mathrm{CI})$ values; and (6) articles in which the statistical methodology included only a univariate analysis that was not verified by a multivariate logistic regression.

\section{Data Extraction and Management}

Two researchers systematically screened the literature, extracted the data, ascertained the type of research, and submitted any objections to a third party for arbitration. The following data were extracted: title, author, year, country, standard of diagnosis, sample size, mean age (expressed as the mean \pm standard deviation if available), sex ratio, and positive dietary and lifestyle risk factors (expressed as ORs or RRs and 95\% CIs). For quantitative variables or grading variables, we only extracted the extreme values as comparators (such as maximum intake vs minimum intake or minimum intake vs maximum intake); for RE, we did not distinguish whether symptoms were present, and for $\mathrm{BE}$, we did not distinguish whether dysplasia was present. The control group was asymptomatic or a population control. Because diet and lifestyle can be expressed in different ways, we classified these factors based on the original text and their intrinsic similarities. 


\section{Results}

\section{General Literature Characteristics}

Finally, 72 articles were included. The specific reasons for exclusion and the numbers of articles excluded are shown in Figure 1. Among the included articles, ten studies were conducted in Western countries (26 articles in total), including Albania, ${ }^{8,9}$ Ireland, ${ }^{10-16}$ Australia, ${ }^{17,18}$ Poland, ${ }^{19,20}$ the Netherlands, ${ }^{21}$ Canada, ${ }^{22}$ the United States, ${ }^{23-29}$ Norway, $^{30}$ Italy, ${ }^{31,32}$ and the UK; ${ }^{33}$ and nine studies were conducted in Eastern countries (46 articles in total), including Pakistan, ${ }^{34}$ South Korea, ${ }^{35-38}$ Malaysia, ${ }^{39}$ Bangladesh, ${ }^{40}$ Japan, ${ }^{41-46}$ Saudi Arabia, ${ }^{47}$ Iran, ${ }^{48}$ India, ${ }^{49-53}$ and China. ${ }^{54-79}$ There were 4 cohort studies, 27 case-control studies, and 41 cross-sectional studies.

Regarding the classification of the related factors, we categorized 20 items related to diet and 11 items related to lifestyle. Then, according to the article, we classified the participants as having GERD, NERD, RE or BE. The details of each article are shown in Appendix 2, and the diet and lifestyle factors in different regions related to each disease type are shown in Table 1.

\section{Dietary and Lifestyle Factors Related to GERD}

In total, 42 articles described the relevant factors associated with GERD; of these studies, eight studies were conducted in Western countries, 34 studies were conducted in Eastern countries, 1 study was a cohort study, 9 studies were case-control studies, and 32 studies were crosssectional studies. The sample size was 173,132 , including 94,235 men and 78,897 women. The main findings related to GERD across the articles are shown in Table 2. The details of the factors are shown in Appendix 3.

Diet and GERD: A vegetarian diet was negatively correlated with GERD. Protein and fat were positively correlated with GERD, and tea was positively related to GERD, except for one article $^{62}$ that showed that greasy food and strong tea were negatively related to GERD. Coffee was negatively related to GERD; soft drinks and infrequent milk intake were positively related to GERD; fruits, citrus, and vitamin $\mathrm{C}$ were negatively related to GERD; and citrus intake between meals, sweet and spicy foods and poor eating habits were positively related to GERD. Sitting or walking after a meal instead of lying down was negatively correlated with GERD, and an interval of less than 3 hours between dinner and sleep was positively correlated with GERD.

The following lifestyle factors are associated with GERD: smoking, alcohol consumption, mental status, higher education, less sleep time, sedentary and physical occupational activities, night work, less exercise, and increased abdominal pressure are positively correlated

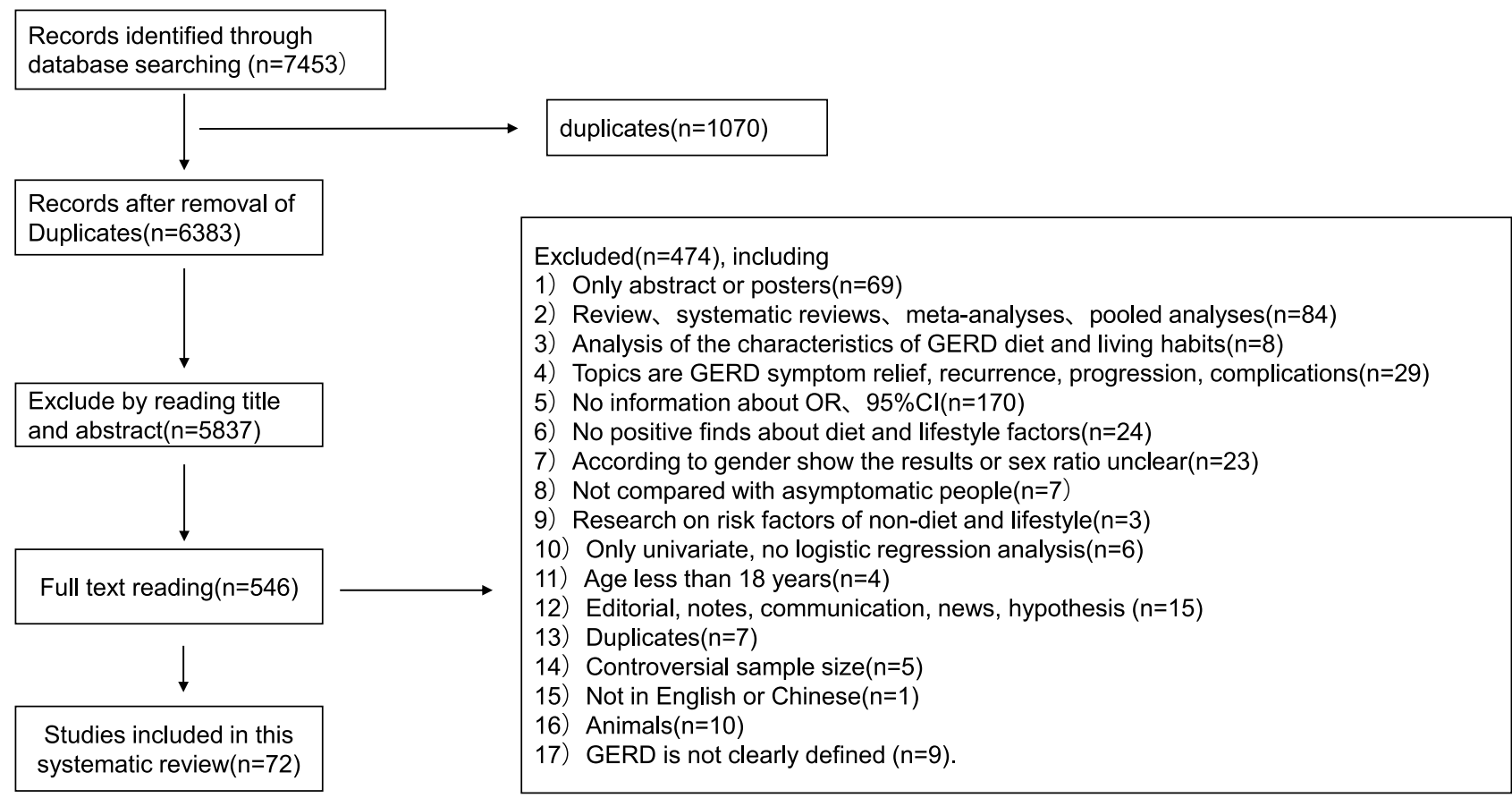

Figure I The flow diagram of the systematic review. 
Table I Diet and Lifestyle Factors Related to Each Disease Type in Western and Eastern Country

\begin{tabular}{|c|c|c|c|c|c|c|c|c|c|}
\hline \multirow{2}{*}{$\begin{array}{l}\text { Diet and } \\
\text { Lifestyle } \\
\text { Categories }\end{array}$} & \multirow[t]{2}{*}{ Details About Each Category } & \multicolumn{2}{|c|}{ GERD } & \multicolumn{2}{|c|}{ NERD } & \multicolumn{2}{|l|}{ RE } & \multicolumn{2}{|c|}{ BE } \\
\hline & & $\mathbf{w}$ & $\mathbf{E}$ & $\mathbf{w}$ & $\mathbf{E}$ & $\mathbf{W}$ & $\mathbf{E}$ & w & E \\
\hline Dietary pattern & Non-Mediterranean diet, consumption of non-vegetarian food, no intake of meat & + & + & & & & & & \\
\hline Dietary habits & $\begin{array}{l}\text { Habit of midnight snacks, skipping breakfast, eating quickly, eating beyond fullness, } \\
\text { eating very hot foods, frequent liquid food consumption }\end{array}$ & & + & & + & & + & & \\
\hline $\begin{array}{l}\text { Habits after } \\
\text { a meal }\end{array}$ & $\begin{array}{l}\text { Lying down soon after eating, Post dinner physical activity (walking), Post dinner } \\
\text { physical activity (lying), Post dinner physical activity (sitting) }\end{array}$ & & + & & & & & & \\
\hline Coffee & Coffee, former coffee drinkers & + & & & & & + & + & \\
\hline Tea & Tea, peppermint tea, green tea, lower intake of salt tea, Tibetan sweet tea & + & + & & + & & + & & + \\
\hline Beverage & Soft drink & + & & & & & & & \\
\hline Milk & Milk, infrequent milk intake & & + & & + & & & & \\
\hline Vegetables & Vegetable, dark green vegetable, bean, vegetable and fruit & & + & & + & & & + & \\
\hline Fruit & Fruit, Citrus intake Between meals, citrus & & & & + & & & + & \\
\hline Vitamins & $\begin{array}{l}\text { Vitamin-E, vitamin-C, folate, vitamin-B2, vitamin-B6, vitamin-BI2, isoflavones, } \\
\text { lutein, anthocyanidins }\end{array}$ & + & & & & + & & + & \\
\hline $\begin{array}{l}\text { Antioxidant } \\
\text { correlation }\end{array}$ & Dietary inflammatory index & & & & & & & + & \\
\hline Micronutrient & Magnesium, dietary iron intake & & & & & + & & + & \\
\hline Salt & Salt intake, extra salt on regular meals, Table salt use & + & + & & & & & & \\
\hline $\begin{array}{l}\text { Taste and flavour } \\
\text { of food }\end{array}$ & Raw or cold food, spicy, sweet & & + & & + & & & & \\
\hline Staple food & Bread high in dietary fibre content, starch & + & & & & + & & & \\
\hline Protein & Processed meat, Tibetan dried meat, egg, Daily meat, fish, and egg intake & & + & & + & + & & & \\
\hline Fat & Fried food, greasy food, high fat diet, saturated fat, monounsaturated fat, total fat & + & + & & + & + & & & + \\
\hline Fiber & Total fiber & & & & & & & + & \\
\hline Energy & Total energy & & & & + & & + & & \\
\hline $\begin{array}{l}\text { Dinner to } \\
\text { bedtime interval }\end{array}$ & $\begin{array}{l}\text { Dinner within two hours before going to sleep, eat } 2 \mathrm{~h} \text { before bed, dinner-to-bed } \\
\text { time was less than } 3 \mathrm{~h}\end{array}$ & & + & & & & & & \\
\hline Smoking related & Smoking, Current smoker, Former smoker & + & + & + & + & & + & + & \\
\hline Drinking related & Alcohol, liquor, wine, beer, alcohol-abusing, Tibetan barley wine & + & + & + & + & + & + & + & + \\
\hline $\begin{array}{l}\text { Psychological } \\
\text { status }\end{array}$ & $\begin{array}{l}\text { Depression, state anxiety, trait anxiety, anxiety, anxiety or depression, strong } \\
\text { psychological stress, poor mental state }\end{array}$ & & + & & + & & + & & \\
\hline Marriage state & Divorced/widowed, widowed/widowers, married & & + & & + & & + & & \\
\hline Education & Education level only elementary, level of education, education level & + & + & & & & & & \\
\hline $\begin{array}{l}\text { Occupation } \\
\text { related traits }\end{array}$ & Sedentary lifestyle, standing occupations, physical labour, night shift & & + & & & & & + & \\
\hline
\end{tabular}

(Continued) 
Table I (Continued).

\begin{tabular}{|c|c|c|c|c|c|c|c|c|c|}
\hline \multirow{2}{*}{$\begin{array}{l}\text { Diet and } \\
\text { Lifestyle } \\
\text { Categories }\end{array}$} & \multirow[t]{2}{*}{ Details About Each Category } & \multicolumn{2}{|c|}{ GERD } & \multicolumn{2}{|c|}{ NERD } & \multicolumn{2}{|l|}{ RE } & \multicolumn{2}{|l|}{ BE } \\
\hline & & $\mathbf{w}$ & $\mathbf{E}$ & $\mathbf{w}$ & $\mathbf{E}$ & $\mathbf{w}$ & E & $\mathbf{w}$ & E \\
\hline $\begin{array}{l}\text { Abdominal } \\
\text { pressure related }\end{array}$ & The belt too tight, wearing girdles or corsets, constipation & & + & & + & & & & \\
\hline Exercise & $\begin{array}{l}\text { Never exercise, physical inactivity, Exercise time less than } 30 \text { minutes, exercise, } \\
\text { exercise daily }>30 \text { minutes, Physical exercise of }>30 \text { minutes }(>3 \text { times/week) }\end{array}$ & + & + & & & & & & \\
\hline Sleep & Insomnia, hours of sleep, staying up late & & + & & & & & & \\
\hline $\begin{array}{l}\text { Living } \\
\text { environment }\end{array}$ & Altitude of residence, length of residence, rural, urban dwelling & & + & & & & & & \\
\hline Others & Snore, Pan masala chewing & & + & & + & & & & \\
\hline
\end{tabular}

Notes: + , stand as there are positive found in here.

Abbreviations: GERD, gastroesophageal reflux disease; NERD, nonerosive gastroesophageal reflux disease; RE, reflux esophagitis; BE, Barrett's esophagus; W, Western country; E, Eastern country.

with GERD. However, drinking beer is negatively correlated with GERD, while altitude, length of residence in the living environment, and urban or rural areas are positively correlated with GERD. Married, divorced, and widowed marital statuses are positively correlated with GERD.

\section{Diet and Lifestyle Factors Related to NERD}

Six articles discussed NERD-related dietary and lifestyle factors. All included studies were conducted in 6 nonEuropean and American countries, 2 studies were casecontrol studies, and 4 studies were cross-sectional studies. The total sample size was 34,762 , including 20,778 males and 13,984 females. There were 1398 confirmed NERD patients. The main findings related to the NERD across articles are shown in Table 3. The details of the factors are shown in Appendix 4.

Diet and NERD: Poor eating habits, including snacking at night and overeating, are positively associated with NERD. The consumption of vegetables, fruits, milk, and tea is negatively correlated with NERD, and the relationship between eggs and NERD is inconsistent. Raw, cold, or spicy food, fat, and high-calorie foods are positively related to NERD.

Lifestyle and NERD: Smoking, alcohol consumption, a poor mental state, insomnia, increased abdominal pressure, and snoring are positively correlated with NERD. Married status and NERD are negatively correlated.

\section{Dietary and Lifestyle Factors Related to RE}

Fifteen articles discussed the dietary and lifestyle risk factors for RE; 7 studies were conducted in European and American countries, 8 studies were conducted in nonEuropean countries, 1 study was a cohort study, 7 studies were case-control studies, and 7 studies were crosssectional studies. The total sample size was 60,718 . There were 38,104 males, 22,614 females, and 1438 confirmed RE patients (with or without symptoms). The main findings related to RE across the articles are shown in Table 4. The details of the factors are shown in Appendix 5.

Diet and RE: Frequent consumption of liquids, coffee, and strong tea is positively correlated with RE; vitamins and micronutrients are negatively correlated with RE; and starch, protein, fat, and energy intake are positively correlated with RE.

Lifestyle and RE: Smoking, alcohol consumption, and a poor mental state are positively correlated with RE, but wine is negatively correlated with RE. Regarding marital status, both married and divorced statuses are positively correlated with RE.

\section{Dietary and Lifestyle Factors Related to $\mathrm{BE}$}

Eighteen articles discussed the diet and lifestyle risk factors for BE; 15 studies were conducted in European and 
Table 2 The Main Findings Related to GERD Across the Articles

\begin{tabular}{|c|c|c|c|c|c|c|}
\hline $\begin{array}{l}\text { First Author, } \\
\text { Publication Year }\end{array}$ & $\begin{array}{l}\text { Area } \\
\text { (Country) }\end{array}$ & $\begin{array}{l}\text { Clinical Study } \\
\text { Design }\end{array}$ & $\begin{array}{l}\text { Positive Related } \\
\text { Factors }\end{array}$ & AOR $(95 \% \mathrm{Cl})$ & $\begin{array}{l}\text { Negative Related } \\
\text { Factors }\end{array}$ & AOR $(95 \% \mathrm{Cl})$ \\
\hline I. Mone, $2016^{8}$ & $\begin{array}{l}\text { Western country } \\
\text { (Albanian) }\end{array}$ & $\begin{array}{l}\text { Cross-sectional } \\
\text { study }\end{array}$ & Non-Mediterranean diet & $2.3(1.2-4.5)$ & & \\
\hline \multirow[t]{4}{*}{ Lulzim Çela, $2013^{9}$} & \multirow{4}{*}{$\begin{array}{l}\text { Western country } \\
\text { (Albanian) }\end{array}$} & \multirow{4}{*}{$\begin{array}{l}\text { Cross-sectional } \\
\text { study }\end{array}$} & Current smoker & $29.3(13.9-61.2)$ & & \\
\hline & & & Former smoker & $9.79(4.22-22.7)$ & & \\
\hline & & & Fried food & $3.01(1.52-6.20)$ & & \\
\hline & & & Physical inactivity & $7.03(2.68-18.4)$ & & \\
\hline \multirow[t]{3}{*}{ Ai Kubo, $2014^{23}$} & \multirow{3}{*}{$\begin{array}{l}\text { Western country } \\
\text { (American) }\end{array}$} & \multirow{3}{*}{$\begin{array}{l}\text { Cross-sectional } \\
\text { study }\end{array}$} & Soft drinks & $1.86(1.16-2.97)$ & Beer & $0.54(0.3 \mathrm{I}-0.96)$ \\
\hline & & & Tea & $1.86(1.02-3.4)$ & \multirow[t]{2}{*}{ Citrus } & \multirow[t]{2}{*}{$0.62(0.4 I-0.94)$} \\
\hline & & & Total fat & $1.77(1.07-2.93)$ & & \\
\hline $\begin{array}{l}\text { Frank } \\
\text { K. Friedenberg, } \\
20 \mathrm{II}^{28}\end{array}$ & $\begin{array}{l}\text { Western country } \\
\text { (American) }\end{array}$ & $\begin{array}{l}\text { Cross-sectional } \\
\text { study }\end{array}$ & Current smoker & $1.74(1.15-2.65)$ & & \\
\hline \multirow[t]{2}{*}{ Gawon Ju, $2013^{36}$} & \multirow{2}{*}{$\begin{array}{l}\text { Eastern country } \\
\text { (Korea) }\end{array}$} & \multirow{2}{*}{$\begin{array}{l}\text { Cross-sectional } \\
\text { study }\end{array}$} & Poor sleep quality & $3.5(1.3-9.3)$ & & \\
\hline & & & Depressed mood & $2.8(1.5-5.3)$ & & \\
\hline $\begin{array}{l}\text { Yasuhiro Fujiwara, } \\
2005^{41}\end{array}$ & $\begin{array}{l}\text { Eastern country } \\
\text { (Japan) }\end{array}$ & $\begin{array}{l}\text { Case control } \\
\text { study }\end{array}$ & $\begin{array}{l}\text { Dinner-to-bed time was } \\
\text { less than } 3 \mathrm{~h}\end{array}$ & $7.45(3.38-16.4)$ & & \\
\hline \multirow{2}{*}{$\begin{array}{l}\text { Tetsuya Murao, } \\
2011^{43}\end{array}$} & \multirow{2}{*}{$\begin{array}{l}\text { Eastern country } \\
\text { (Japan) }\end{array}$} & \multirow{2}{*}{$\begin{array}{l}\text { Cross-sectional } \\
\text { study }\end{array}$} & \multirow[t]{2}{*}{ Green tea drinker } & \multirow[t]{2}{*}{$1.44(1.07-1.94)$} & Hours of sleep & $0.90(0.82-0.99)$ \\
\hline & & & & & Exercise & $0.74(0.61-0.89)$ \\
\hline $\begin{array}{l}\text { Masaki Miyamoto, } \\
2007^{44}\end{array}$ & $\begin{array}{l}\text { Eastern country } \\
\text { (Japan) }\end{array}$ & Cohort study & Constipation & $7.259(2.623-20.092)$ & & \\
\hline \multirow[t]{2}{*}{$\begin{array}{l}\text { Maria Pina Dore, } \\
2008^{32}\end{array}$} & \multirow[t]{2}{*}{$\begin{array}{l}\text { Western country } \\
\text { (Italy) }\end{array}$} & \multirow[t]{2}{*}{$\begin{array}{l}\text { Case control } \\
\text { study }\end{array}$} & $\begin{array}{l}\text { Education level only } \\
\text { elementary }\end{array}$ & $3.2(1.8-5.5)$ & & \\
\hline & & & Never exercise & $1.9(1.2-3.5)$ & & \\
\hline $\begin{array}{l}\text { Mirosław Jarosz, } \\
2014^{20}\end{array}$ & $\begin{array}{l}\text { Western country } \\
\text { (Australia) }\end{array}$ & $\begin{array}{l}\text { Case control } \\
\text { study }\end{array}$ & Peppermint tea & $2.00(1.08-3.70)$ & & \\
\hline $\begin{array}{l}\text { Omid Eslami, } \\
2017^{48}\end{array}$ & $\begin{array}{l}\text { Eastern country } \\
\text { (Iran) }\end{array}$ & $\begin{array}{l}\text { Cross-sectional } \\
\text { study }\end{array}$ & $\begin{array}{l}\text { Citrus intake Between } \\
\text { meals }\end{array}$ & $2.22(1.3-3.81)$ & $\geq 12$ years schooling & $0.55(0.33-0.91)$ \\
\hline \multirow[t]{7}{*}{$\begin{array}{l}\text { Haoxiang Zhang, } \\
2019^{54}\end{array}$} & \multirow[t]{7}{*}{$\begin{array}{l}\text { Eastern country } \\
\text { (China) }\end{array}$} & \multirow[t]{7}{*}{$\begin{array}{l}\text { Cross-sectional } \\
\text { study }\end{array}$} & $\begin{array}{l}\text { Education level (high } \\
\text { school vs primary) }\end{array}$ & $2.804(2.090-3.761)$ & & \\
\hline & & & Altitude of residence & $2.469(1.714-3.556)$ & & \\
\hline & & & Length of residence & $2.218(1.836-2.679)$ & & \\
\hline & & & Tibetan sweet tea & $2.158(1.782-2.613)$ & & \\
\hline & & & Tibetan barley wine & $1.271(1.060-1.523)$ & & \\
\hline & & & Tibetan dried meat & $1.278(1.067-1.532)$ & & \\
\hline & & & Staying up late & $1.229(1.026-1.472)$ & & \\
\hline Jia $\mathrm{He}, 2010^{56}$ & $\begin{array}{l}\text { Eastern country } \\
\text { (China) }\end{array}$ & $\begin{array}{l}\text { Cross-sectional } \\
\text { study }\end{array}$ & Rural & $1.40(1.13-1.72)$ & & \\
\hline
\end{tabular}

(Continued) 
Table 2 (Continued).

\begin{tabular}{|c|c|c|c|c|c|c|}
\hline $\begin{array}{l}\text { First Author, } \\
\text { Publication Year }\end{array}$ & $\begin{array}{l}\text { Area } \\
\text { (Country) }\end{array}$ & $\begin{array}{l}\text { Clinical Study } \\
\text { Design }\end{array}$ & $\begin{array}{l}\text { Positive Related } \\
\text { Factors }\end{array}$ & AOR $(95 \% \mathrm{Cl})$ & $\begin{array}{l}\text { Negative Related } \\
\text { Factors }\end{array}$ & AOR $(95 \% \mathrm{Cl})$ \\
\hline X.-Q. Ma, $2009^{61}$ & $\begin{array}{l}\text { Eastern country } \\
\text { (China) }\end{array}$ & $\begin{array}{l}\text { Cross-sectional } \\
\text { study }\end{array}$ & Urban dwelling & $3.6(1.2-10.4)$ & & \\
\hline \multirow[t]{3}{*}{ Yan Gong, $2019^{57}$} & \multirow{3}{*}{$\begin{array}{l}\text { Eastern country } \\
\text { (China) }\end{array}$} & \multirow{3}{*}{$\begin{array}{l}\text { Cross-sectional } \\
\text { study }\end{array}$} & Smoking & $1.19(1.12-1.264)$ & Salt intake & $0.903(0.853-0.956)$ \\
\hline & & & Alcohol consumption & $1.278(1.207-1.353)$ & Physical activity & $0.846(0.808-0.886)$ \\
\hline & & & $\begin{array}{l}\text { Daily meat, fish, and egg } \\
\text { intake }\end{array}$ & $1.088(1.042-1.135)$ & Daily fruit intake & $0.91(0.856-0.967)$ \\
\hline \multirow{6}{*}{$\begin{array}{l}\text { Ling-Zhi Yuan, } \\
2019^{58}\end{array}$} & \multirow{6}{*}{$\begin{array}{l}\text { Eastern country } \\
\text { (China) }\end{array}$} & \multirow{6}{*}{$\begin{array}{l}\text { Cross-sectional } \\
\text { study }\end{array}$} & Eating quickly & $4.06(3.11-5.29)$ & & \\
\hline & & & Eating beyond fullness & $2.85(2.18-3.73)$ & & \\
\hline & & & Wearing girdles or corsets & $2.19(1.42-3.38)$ & & \\
\hline & & & Eating very hot foods & $1.54(1.16-2.05)$ & & \\
\hline & & & $\begin{array}{l}\text { Lying down soon after } \\
\text { eating }\end{array}$ & I.81 (1.37-2.4) & & \\
\hline & & & Smoking & $1.52(1.07-2.15)$ & & \\
\hline \multirow[t]{2}{*}{ Juan Du, $2007^{62}$} & \multirow{2}{*}{$\begin{array}{l}\text { Eastern country } \\
\text { (China) }\end{array}$} & \multirow{2}{*}{$\begin{array}{l}\text { Cross-sectional } \\
\text { study }\end{array}$} & \multirow[t]{2}{*}{ Divorced/widowed } & \multirow[t]{2}{*}{$1.82(1.27-2.60)$} & Greasy food & $0.75(0.60-0.95)$ \\
\hline & & & & & Strong tea drinking & $0.67(0.50-0.89)$ \\
\hline \multirow[t]{3}{*}{ M Nilsson, $2004^{30}$} & \multirow[t]{3}{*}{$\begin{array}{l}\text { Western country } \\
\text { (Norway) }\end{array}$} & \multirow[t]{3}{*}{$\begin{array}{l}\text { Case Control } \\
\text { study }\end{array}$} & $\begin{array}{l}\text { Smoked daily for more } \\
\text { than } 20 \text { years }\end{array}$ & $1.7(1.5-1.9)$ & Coffee & $0.6(0.4-0.7)$ \\
\hline & & & Table salt use & $1.5(1.2-1.8)$ & $\begin{array}{l}\text { Bread high in dietary } \\
\text { fibre content }\end{array}$ & $0.5(0.4-0.7)$ \\
\hline & & & Extra salt on regular meals & $1.7(1.4-2.0)$ & $\begin{array}{l}\text { Physical exercise of }>30 \\
\text { minutes ( }>3 / \text { week) }\end{array}$ & $0.7(0.6-0.9)$ \\
\hline \multirow[t]{2}{*}{$\begin{array}{l}\text { Sushil Kumar, } \\
2011^{49}\end{array}$} & \multirow[t]{2}{*}{$\begin{array}{l}\text { Eastern country } \\
\text { (India) }\end{array}$} & \multirow[t]{2}{*}{$\begin{array}{l}\text { Cross-sectional } \\
\text { study }\end{array}$} & Sedentary lifestyle & $2.786(1.016-7.638)$ & $\begin{array}{l}\text { Intake of fresh fruits } \geq 1 / \\
\text { week }\end{array}$ & $0.631(0.409-0.973)$ \\
\hline & & & Lower intake of salt tea & $1.663(1.014-2.726)$ & No intake of meat & $0.84 \mid(0.715-0.990)$ \\
\hline \multirow{2}{*}{$\begin{array}{l}\text { Sudipta Dhar } \\
\text { Chowdhury, } \\
2019^{50}\end{array}$} & \multirow{2}{*}{$\begin{array}{l}\text { Eastern country } \\
\text { (India) }\end{array}$} & \multirow{2}{*}{$\begin{array}{l}\text { Cross-sectional } \\
\text { study }\end{array}$} & Living in urban area & $2.3(1.9-2.8)$ & & \\
\hline & & & Infrequent milk intake & $1.6(1.3-1.9)$ & & \\
\hline \multirow{2}{*}{$\begin{array}{l}\text { Hai-Yun Wang, } \\
2016^{51}\end{array}$} & \multirow{2}{*}{$\begin{array}{l}\text { Eastern country } \\
\text { (India) }\end{array}$} & \multirow{2}{*}{$\begin{array}{l}\text { Cross-sectional } \\
\text { study }\end{array}$} & Urban & $1.8(1.3-2.5)$ & & \\
\hline & & & Pan masala chewing & $2.0(1.2-3.2)$ & & \\
\hline $\begin{array}{l}\text { Praveen Kumar } \\
\text { Sharma, } 2010^{52}\end{array}$ & $\begin{array}{l}\text { Eastern country } \\
\text { (India) }\end{array}$ & $\begin{array}{l}\text { Cross-sectional } \\
\text { study }\end{array}$ & Current smoking & $1.48(1.19-1.83)$ & & \\
\hline $\begin{array}{l}\text { Shobna J. Bhatia, } \\
2011^{53}\end{array}$ & $\begin{array}{l}\text { Eastern country } \\
\text { (India) }\end{array}$ & $\begin{array}{l}\text { Cross-sectional } \\
\text { study }\end{array}$ & & & $\begin{array}{l}\text { Consumption of non- } \\
\text { vegetarian food }\end{array}$ & $0.34(0.211-0.545)$ \\
\hline
\end{tabular}

(Continued) 
Table 2 (Continued).

\begin{tabular}{|c|c|c|c|c|c|c|}
\hline $\begin{array}{l}\text { First Author, } \\
\text { Publication Year }\end{array}$ & $\begin{array}{l}\text { Area } \\
\text { (Country) }\end{array}$ & $\begin{array}{l}\text { Clinical Study } \\
\text { Design }\end{array}$ & $\begin{array}{l}\text { Positive Related } \\
\text { Factors }\end{array}$ & AOR (95\% Cl) & $\begin{array}{l}\text { Negative Related } \\
\text { Factors }\end{array}$ & AOR $(95 \% \mathrm{CI})$ \\
\hline \multirow[t]{7}{*}{$\begin{array}{l}\text { Shahid Ahmed, } \\
2020^{34}\end{array}$} & \multirow[t]{7}{*}{$\begin{array}{l}\text { Eastern country } \\
\text { (Pakistan) }\end{array}$} & \multirow[t]{7}{*}{$\begin{array}{l}\text { Cross-sectional } \\
\text { study }\end{array}$} & $\begin{array}{l}\text { Exercise time less than } 30 \\
\text { minutes }\end{array}$ & $6.47(4.91-8.53)$ & $\begin{array}{l}\text { Post dinner physical } \\
\text { activity (walking) }\end{array}$ & $0.25(0.13-0.47)$ \\
\hline & & & Habit of midnight snacks & $5.08(4.03-6.4)$ & $\begin{array}{l}\text { Post dinner physical } \\
\text { activity (sitting) }\end{array}$ & $0.45(0.24-0.84)$ \\
\hline & & & $\begin{array}{l}\text { Feeling of inadequate } \\
\text { sleep }\end{array}$ & $3.22(2.57-4.03)$ & \multirow[t]{5}{*}{$\begin{array}{l}\text { Exercise daily }>30 \\
\text { minutes }\end{array}$} & \multirow[t]{5}{*}{$0.02(0.01-0.03)$} \\
\hline & & & $\begin{array}{l}\text { Frequent skipping } \\
\text { breakfast }\end{array}$ & $7(2.17-3.35)$ & & \\
\hline & & & $\begin{array}{l}\text { Dinner within two hours } \\
\text { before going to sleep }\end{array}$ & $6.98(5.36-9.08)$ & & \\
\hline & & & Habit of smoking & $6.25(4.4-8.91)$ & & \\
\hline & & & $\begin{array}{l}\text { Post dinner physical } \\
\text { activity (lying) }\end{array}$ & $2.24(1.19-4.2)$ & & \\
\hline \multirow[t]{3}{*}{ Shaha $M, 2012^{40}$} & \multirow[t]{3}{*}{$\begin{array}{l}\text { Eastern country } \\
\text { (Bangladesh) }\end{array}$} & \multirow[t]{3}{*}{$\begin{array}{l}\text { Cross-sectional } \\
\text { study }\end{array}$} & $\begin{array}{l}\text { Level of education } \\
\text { (Primary) }\end{array}$ & $3.095(1.5 \mathrm{II}-25.889)$ & & \\
\hline & & & Married & $4.852(2.23-10.553)$ & & \\
\hline & & & Widowed/widowers & $14.6(5.879-36.258)$ & & \\
\hline $\begin{array}{l}\text { P. J. Veugelers, } \\
2006^{22}\end{array}$ & $\begin{array}{l}\text { Western country } \\
\text { (Canada) }\end{array}$ & $\begin{array}{l}\text { Case control } \\
\text { study }\end{array}$ & Liquor & $2.69(1.05-6.92)$ & Vitamin C & $0.4(0.19-0.87)$ \\
\hline $\begin{array}{l}\text { Nabil Joseph } \\
\text { Awadalla, 201947 }\end{array}$ & $\begin{array}{l}\text { Eastern country } \\
\text { (Saudi Arabia) }\end{array}$ & $\begin{array}{l}\text { Cross-sectional } \\
\text { study }\end{array}$ & Insomnia & $1.65(1.36-2.01)$ & & \\
\hline \multirow{2}{*}{$\begin{array}{l}\text { Modh Said Rosaida, } \\
2004^{39}\end{array}$} & \multirow{2}{*}{$\begin{array}{l}\text { Eastern country } \\
\text { (Malaysia) }\end{array}$} & \multirow{2}{*}{$\begin{array}{l}\text { Cross-sectional } \\
\text { study }\end{array}$} & Alcohol & $2.42(1.11-5.23)$ & & \\
\hline & & & Education level & $1.52(1.02-2.26)$ & & \\
\hline \multirow{2}{*}{$\begin{array}{l}\text { ZHANG Min, } \\
2018^{63}\end{array}$} & \multirow{2}{*}{$\begin{array}{l}\text { Eastern country } \\
\text { (China) }\end{array}$} & \multirow{2}{*}{$\begin{array}{l}\text { Cross-sectional } \\
\text { study }\end{array}$} & Greasy food & 1.794 (I.237 2.73I) & & \\
\hline & & & Smoking & $2.07 \mid(1.560 \sim 2.869)$ & & \\
\hline \multirow{6}{*}{$\begin{array}{l}\text { YAO XiaoJun, } \\
2018^{64}\end{array}$} & \multirow{6}{*}{$\begin{array}{l}\text { Eastern country } \\
\text { (China) }\end{array}$} & \multirow{6}{*}{$\begin{array}{l}\text { Cross-sectional } \\
\text { study }\end{array}$} & Eating beyond fullness & $3.2(2.13 \mid-5.042)$ & & \\
\hline & & & High fat diet & 7.568, (4.557-8.908) & & \\
\hline & & & Liquor & $2.262(1.87 \mid-4.322)$ & & \\
\hline & & & Mental stress & $2.122(1.551-4.072)$ & & \\
\hline & & & Constipation & $2.329(1.409-4.27 I)$ & & \\
\hline & & & Sweet & $1.952(1.121-3.122)$ & & \\
\hline \multirow{2}{*}{$\begin{array}{l}\text { JIANG Chenglin, } \\
2016^{66}\end{array}$} & \multirow{2}{*}{$\begin{array}{l}\text { Eastern country } \\
\text { (China) }\end{array}$} & \multirow{2}{*}{$\begin{array}{l}\text { Cross-sectional } \\
\text { study }\end{array}$} & Strong tea & 2. $145(1.338-3.438)$ & & \\
\hline & & & Greasy food & 2. $016(1.208-3.318)$ & & \\
\hline \multirow{4}{*}{$\begin{array}{l}\text { RONG Liang, } \\
2013^{68}\end{array}$} & \multirow{4}{*}{$\begin{array}{l}\text { Eastern country } \\
\text { (China) }\end{array}$} & \multirow{4}{*}{$\begin{array}{l}\text { Cross-sectional } \\
\text { study }\end{array}$} & High fat diet & $7.964(6.146-10.319)$ & & \\
\hline & & & Alcohol & $3.804(2.982-4.852)$ & & \\
\hline & & & Strong tea & $2.758(2.17-3.504)$ & & \\
\hline & & & Eating beyond fullness & $2.408(1.896-3.06)$ & & \\
\hline
\end{tabular}

(Continued) 
Table 2 (Continued).

\begin{tabular}{|c|c|c|c|c|c|c|}
\hline $\begin{array}{l}\text { First Author, } \\
\text { Publication Year }\end{array}$ & $\begin{array}{l}\text { Area } \\
\text { (Country) }\end{array}$ & $\begin{array}{l}\text { Clinical Study } \\
\text { Design }\end{array}$ & $\begin{array}{l}\text { Positive Related } \\
\text { Factors }\end{array}$ & AOR $(95 \% \mathrm{Cl})$ & $\begin{array}{l}\text { Negative Related } \\
\text { Factors }\end{array}$ & AOR $(95 \% \mathrm{Cl})$ \\
\hline \multirow[t]{2}{*}{$\begin{array}{l}\text { CHEN HuiXin, } \\
2006^{72}\end{array}$} & \multirow[t]{2}{*}{$\begin{array}{l}\text { Eastern country } \\
\text { (China) }\end{array}$} & \multirow[t]{2}{*}{$\begin{array}{l}\text { Case control } \\
\text { study }\end{array}$} & $\begin{array}{l}\text { Divorced, separated or } \\
\text { widowed }\end{array}$ & $4.61(2.15-9.89)$ & & \\
\hline & & & Heavy working pressure & $3.43(1.72-6.84)$ & & \\
\hline \multirow[t]{4}{*}{ JIANG Chu, $2010^{69}$} & \multirow{4}{*}{$\begin{array}{l}\text { Eastern country } \\
\text { (China) }\end{array}$} & \multirow{4}{*}{$\begin{array}{l}\text { Cross-sectional } \\
\text { study }\end{array}$} & Rural & $2.237(1.422-3.517)$ & & \\
\hline & & & High educated & $1.242(1.001-1.542)$ & & \\
\hline & & & Work and life are stressful & 1.277 (1.089-1.497) & & \\
\hline & & & Poor mental state & $1.20(1.046-1.665)$ & & \\
\hline \multirow{3}{*}{$\begin{array}{l}\text { SHEN Xu-De, } \\
2010^{70}\end{array}$} & \multirow{3}{*}{$\begin{array}{l}\text { Eastern country } \\
\text { (China) }\end{array}$} & \multirow{3}{*}{$\begin{array}{l}\text { Cross-sectional } \\
\text { study }\end{array}$} & Eating beyond fullness & $2.053(1.293,-3.26)$ & & \\
\hline & & & Sweet & $2.413(1.252-3.679)$ & & \\
\hline & & & Constipation & $1.65(1.038-2.62 I)$ & & \\
\hline \multirow{5}{*}{$\begin{array}{l}\text { LIN XiaoDan, } \\
2018^{73}\end{array}$} & \multirow{5}{*}{$\begin{array}{l}\text { Eastern country } \\
\text { (China) }\end{array}$} & \multirow{5}{*}{$\begin{array}{l}\text { Case control } \\
\text { study }\end{array}$} & Skipping breakfast & 2. $879(1.479-5.605)$ & & \\
\hline & & & Eat $2 \mathrm{~h}$ before bed & 2. $402(1.213-4.756)$ & & \\
\hline & & & Tea & 4.857 (2. 468-9. 559) & & \\
\hline & & & Alcohol & $3.613(1.899-6.874)$ & & \\
\hline & & & Shortage of sleep & $2.832(1.501-5.345)$ & & \\
\hline \multirow{4}{*}{$\begin{array}{l}\text { YUAN LinZHi, } \\
2017^{74}\end{array}$} & \multirow{4}{*}{$\begin{array}{l}\text { Eastern country } \\
\text { (China) }\end{array}$} & \multirow{4}{*}{$\begin{array}{l}\text { Case control } \\
\text { study }\end{array}$} & Eating quickly & $3.214(2.171-4.759)$ & & \\
\hline & & & Eating beyond fullness & $2.936(1.981-4.350)$ & & \\
\hline & & & The belt too tight & $2.003(1.013-3.961)$ & & \\
\hline & & & Eating very hot foods & $1.570(1.044 \sim 2.362)$ & & \\
\hline \multirow{2}{*}{$\begin{array}{l}\text { GAO HongLiang, } \\
2012^{75}\end{array}$} & \multirow{2}{*}{$\begin{array}{l}\text { Eastern country } \\
\text { (China) }\end{array}$} & \multirow{2}{*}{$\begin{array}{l}\text { Case control } \\
\text { study }\end{array}$} & Spicy & $5.469(2.57-11.64)$ & & \\
\hline & & & Constipation & $3.76(1.592-8.884)$ & & \\
\hline \multirow{2}{*}{$\begin{array}{l}\text { YIN Yanwei, } \\
2012^{76}\end{array}$} & \multirow{2}{*}{$\begin{array}{l}\text { Eastern country } \\
\text { (China) }\end{array}$} & \multirow{2}{*}{$\begin{array}{l}\text { Cross-sectional } \\
\text { study }\end{array}$} & Alcohol & $2.65(1.03-6.8 I)$ & & \\
\hline & & & Eating beyond fullness & $2.81(1.04-7.58)$ & & \\
\hline \multirow{2}{*}{$\begin{array}{l}\text { JIANG Xuan, } \\
2011^{77}\end{array}$} & \multirow{2}{*}{$\begin{array}{l}\text { Eastern country } \\
\text { (China) }\end{array}$} & \multirow{2}{*}{$\begin{array}{l}\text { Cross-sectional } \\
\text { study }\end{array}$} & Alcohol & $2.63(1.17-5.92)$ & & \\
\hline & & & Physical work & I. $79(1.13-2.86)$ & & \\
\hline \multirow{4}{*}{$\begin{array}{l}\text { HU ShuiQing, } \\
2009^{78}\end{array}$} & \multirow{4}{*}{$\begin{array}{l}\text { Eastern country } \\
\text { (China) }\end{array}$} & \multirow{4}{*}{$\begin{array}{l}\text { Cross-sectional } \\
\text { study }\end{array}$} & Eating beyond fullness & $2.78(1.76-4.18)$ & & \\
\hline & & & Greasy food & $4.36(2.61-9.08)$ & & \\
\hline & & & Constipation & $2.06(1.18-3.48)$ & & \\
\hline & & & Mental stress & $2.11(1.2-3.52)$. & & \\
\hline
\end{tabular}

(Continued) 
Table 2 (Continued).

\begin{tabular}{|c|c|c|c|c|c|c|}
\hline $\begin{array}{l}\text { First Author, } \\
\text { Publication Year }\end{array}$ & $\begin{array}{l}\text { Area } \\
\text { (Country) }\end{array}$ & $\begin{array}{l}\text { Clinical Study } \\
\text { Design }\end{array}$ & $\begin{array}{l}\text { Positive Related } \\
\text { Factors }\end{array}$ & AOR $(95 \% \mathrm{Cl})$ & $\begin{array}{l}\text { Negative Related } \\
\text { Factors }\end{array}$ & AOR $(95 \% \mathrm{Cl})$ \\
\hline \multirow{7}{*}{$\begin{array}{l}\text { ZHANG Hong, } \\
2007^{79}\end{array}$} & \multirow{7}{*}{$\begin{array}{l}\text { Eastern country } \\
\text { (China) }\end{array}$} & \multirow{7}{*}{$\begin{array}{l}\text { Cross-sectional } \\
\text { study }\end{array}$} & Night shift & $1.381(1.1113-1.713)$ & & \\
\hline & & & Physical labour & $2.043(1.554-2.687)$ & & \\
\hline & & & Eating beyond fullness & $1.775(1.506-2.091)$ & & \\
\hline & & & Greasy food & $1.506(1.269-1.788)$ & & \\
\hline & & & Strong tea & $1.572(1.314-1.88)$ & & \\
\hline & & & Sweet & $1.273(1.075-1.508)$ & & \\
\hline & & & Constipation & $1.724(1.438-2.068)$ & & \\
\hline
\end{tabular}

Abbreviations: GERD, gastroesophageal reflux disease; AOR, adjusted odds ratio.

American countries, 3 studies were conducted in nonEuropean countries, 2 studies were cohort studies, 13 studies were case-control studies, and 3 studies were cross-sectional studies. The sample size was 58,032, including 30,135 males and 27,897 females. Among the participants, 4185 participants were diagnosed with $\mathrm{BE}$ (with or without heterogeneous hyperplasia and symptoms). The main findings related to BE across the articles are shown in Table 5. The details of the factors are shown in Appendix 6.

Diet and BE: Coffee, tea and fruit are positively correlated with $\mathrm{BE}$, and the intake of vegetables, vitamins, micronutrients, and fiber is negatively correlated with $\mathrm{BE}$; however, vitamin $\mathrm{B} 12$ is positively correlated with BE.

Lifestyle and BE: Smoking and alcohol consumption are positively correlated with $\mathrm{BE}$, but wine and beer are negatively correlated with BE. Standing occupational activity was negatively correlated with BE.

\section{Discussion}

In summary, protein and fat as dietary factors and smoking, alcohol consumption (except for beer and wine), and mental state as lifestyle factors were all observed to be positively correlated with GERD and other types of reflux. Vegetarian diets, fruits, vegetables, vitamins and fiber were negatively correlated with GERD and other types of reflux, while poor eating habits were positively correlated with GERD, GER, and NERD. This association was not found in the RE and BE types. We speculate that poor eating habits may aggravate the perception of symptoms of GERD. An interval of less than 3 hours between dinner and sleep or physical exercise was negatively correlated with GERD. Many dietary and lifestyle factors affect the onset of GERD, and the factors influencing the different types vary.

We also found that eating citrus fruits with meals is positively correlated with GERD. ${ }^{48}$ However, it has also been shown that citrus is negatively correlated with GERD, which may be related to the fact that GERD patients avoid eating such foods, which leads to biased results. ${ }^{23}$ Studies have shown that acidic fruits, such as citrus fruits and tomatoes, can induce reflux-related symptoms. The possible reasons are increased acidic fluid intake and frequent swallowing. ${ }^{80-82}$ Moreover, less volume to swallow and acidic beverages cause the $\mathrm{pH}$ of the esophagus to rapidly decrease to $<4$. In vitamin-related studies, vitamins have been shown to be negatively correlated with GERD, RE, and BE, but vitamin B12 was found to be positively correlated with BE. This finding may be explained by vitamin B12 being mainly derived from meat and milk, and vitamin B12 is almost absent from plant foods. ${ }^{83}$

The dietary factors related to fat in this article were fried foods, greasy foods, high-fat diets, saturated fatty acids, monounsaturated fatty acids, and total fats. Studies have shown that dietary fats are mainly used to enhance people's gastroesophageal reaction. The perception of fluid symptoms is thought to be involved rather than increasing the time of esophageal acid exposure and the frequency of TLESR. ${ }^{84}$ Spicy food is positively correlated with GERD and NERD. ${ }^{65,75}$ Capsaicin increases the pressure of the LES, esophageal contraction and transmission speed in healthy subjects, and as spiciness increases, the impact on the esophagus and stomach becomes more significant. ${ }^{85,86}$ 
Table 3 The Main Findings Related to NERD Across the Articles

\begin{tabular}{|c|c|c|c|c|c|c|}
\hline $\begin{array}{l}\text { First } \\
\text { Author, } \\
\text { Publication } \\
\text { Year }\end{array}$ & $\begin{array}{l}\text { Area } \\
\text { (Country) }\end{array}$ & $\begin{array}{l}\text { Clinical Study } \\
\text { Design }\end{array}$ & $\begin{array}{l}\text { Positive Related } \\
\text { Factors }\end{array}$ & AOR (95\% Cl) & $\begin{array}{l}\text { Negative } \\
\text { Related } \\
\text { Factors }\end{array}$ & AOR $(95 \% \mathrm{Cl})$ \\
\hline \multirow{3}{*}{$\begin{array}{l}\text { Nobuyuki } \\
\text { Matsuki, } \\
2013^{45}\end{array}$} & \multirow{3}{*}{$\begin{array}{l}\text { Eastern country } \\
\text { (Japan) }\end{array}$} & \multirow{3}{*}{$\begin{array}{l}\text { Cross-sectional } \\
\text { study }\end{array}$} & Egg & $1.89(1.01-3.5)$ & & \\
\hline & & & Strong psychological stress & $1.77(1.18-2.62)$ & & \\
\hline & & & Sleep shortage & $2.44(1.54-3.88)$ & & \\
\hline \multirow{5}{*}{$\begin{array}{l}\text { Su Youn Nam, } \\
2016^{35}\end{array}$} & \multirow{5}{*}{$\begin{array}{l}\text { Eastern country } \\
\text { (Korea) }\end{array}$} & \multirow{5}{*}{$\begin{array}{l}\text { Cross-sectional } \\
\text { study }\end{array}$} & Current smoker & $1.54(1.29-1.84)$ & Beans & $0.78(0.64-0.95)$ \\
\hline & & & \multirow[t]{4}{*}{ Total energy intake } & \multirow[t]{4}{*}{$1.07(1.0-1.14)$} & Fruits & $0.78(0.64-0.95)$ \\
\hline & & & & & Egg & $0.78(0.64-0.96)$ \\
\hline & & & & & Milk & $0.78(0.65-0.94)$ \\
\hline & & & & & Drink-tea & $0.62(0.5-0.76)$ \\
\hline \multirow{4}{*}{$\begin{array}{l}\text { Ji Min Choi, } \\
2018^{37}\end{array}$} & \multirow{4}{*}{$\begin{array}{l}\text { Eastern country } \\
\text { (Korea) }\end{array}$} & \multirow{4}{*}{$\begin{array}{l}\text { Cross-sectional } \\
\text { study }\end{array}$} & State anxiety & $1.89(1.53-2.33)$ & \multirow[t]{4}{*}{ Marriage status } & \multirow[t]{4}{*}{$0.71(0.58-0.87)$} \\
\hline & & & Rait anxiety & $1.78(1.34-2.35)$ & & \\
\hline & & & Depression & $2.21(1.75-2.8)$ & & \\
\hline & & & Current smoking & 1.37 (1.18-1.59). & & \\
\hline \multirow{2}{*}{$\begin{array}{l}\text { Juan Du, } \\
2007^{62}\end{array}$} & \multirow{2}{*}{$\begin{array}{l}\text { Eastern } \\
\text { country (China) }\end{array}$} & \multirow{2}{*}{$\begin{array}{l}\text { Cross-sectional } \\
\text { study }\end{array}$} & Greasy food & $1.65(1.16-2.36)$ & & \\
\hline & & & Constipation & $1.5 \mathrm{I}(1.0 \mathrm{I}-2.25)$ & & \\
\hline \multirow{4}{*}{$\begin{array}{l}\text { CHEN LiPing, } \\
2016^{71}\end{array}$} & \multirow{4}{*}{$\begin{array}{l}\text { Eastern country } \\
\text { (China) }\end{array}$} & \multirow{4}{*}{$\begin{array}{l}\text { Case control } \\
\text { study }\end{array}$} & Habit of midnight snacks & $2.752(1.449-5.228)$ & & \\
\hline & & & Snore & $2.334(1.361-4.004)$ & & \\
\hline & & & Alcohol & $3.957(1.067-14.673)$ & & \\
\hline & & & Anxiety or depression & $2.723(1.407-5.267)$ & & \\
\hline \multirow{5}{*}{$\begin{array}{l}\text { WANG Yi, } \\
2018^{65}\end{array}$} & \multirow{5}{*}{$\begin{array}{l}\text { Eastern country } \\
\text { (China) }\end{array}$} & \multirow{5}{*}{$\begin{array}{l}\text { Case control } \\
\text { study }\end{array}$} & Raw or cold food & $5.47(I .2 I-24.7 I)$ & & \\
\hline & & & Spicy & $3.36(1.15-9.84)$ & & \\
\hline & & & Eating beyond fullness & $9.98(3.57-27.88)$ & & \\
\hline & & & Anxiety & $4.09(1.8-9.26)$ & & \\
\hline & & & Depression & $2.21(1.25-3.9)$ & & \\
\hline
\end{tabular}

Abbreviations: NERD, nonerosive gastroesophageal reflux disease; AOR, adjusted odds ratio.

Regarding eating habits, eating snacks at night, frequently skipping breakfast, eating quickly, eating hot food, and overeating are positively correlated with GERD, ${ }^{34,58,64,68,70,73,74,76,78,79}$ and snacking at night and overeating are positively correlated with NERD. ${ }^{65,71}$ Frequent liquid consumption is positively correlated with RE. ${ }^{55} \mathrm{We}$ reasonably speculate that appropriate, moderate and regular eating habits are important factors for preventing GERD.

Regarding postmeal habits, sitting or walking after a meal instead of lying down is beneficial for patients with gastroesophageal reflux disease. It is inferred that a postprandial posture is a factor influencing GERD. However, studies have shown that body position does not impact the changes in gastric acidity that occur in healthy subjects after fasting or after meals. ${ }^{87}$ In one study, 24hour esophageal $\mathrm{pH}$ monitoring was used to compare subjects with esophagitis with bidirectional reflux and subjects with only upright reflux without esophagitis, and it was found that the subjects with bidirectional reflux and esophagitis experienced increased reflux in the supine 
Table 4 The Main Findings Related to RE Across the Articles

\begin{tabular}{|c|c|c|c|c|c|c|}
\hline $\begin{array}{l}\text { First Author, } \\
\text { Publication } \\
\text { Year }\end{array}$ & Area (Country) & $\begin{array}{l}\text { Clinical Study } \\
\text { Design }\end{array}$ & Positive Related Factors & AOR (95\% Cl) & $\begin{array}{l}\text { Negative Related } \\
\text { Factors }\end{array}$ & AOR $(95 \% \mathrm{Cl})$ \\
\hline $\begin{array}{l}\text { Anna } \\
\text { Boguradzka, } \\
2011^{19}\end{array}$ & $\begin{array}{l}\text { Western country } \\
\text { (Australia) }\end{array}$ & $\begin{array}{l}\text { Cross-sectional } \\
\text { study }\end{array}$ & Alcohol-abusing & $7.34(2.27-23.7)$ & & \\
\hline \multirow{3}{*}{$\begin{array}{l}\text { Su Youn Nam, } \\
2016^{35}\end{array}$} & \multirow{3}{*}{$\begin{array}{l}\text { Eastern country } \\
\text { (Korea) }\end{array}$} & \multirow{3}{*}{$\begin{array}{l}\text { Cross-sectional } \\
\text { study }\end{array}$} & Current smoker & $1.7(1.44-2.01)$ & & \\
\hline & & & Current drinker & $1.26(1.03-1.54)$ & & \\
\hline & & & Total energy. & $1.08(1.0-1.16)$ & & \\
\hline \multirow{3}{*}{$\begin{array}{l}\text { Ji Min Choi, } \\
2018^{37}\end{array}$} & \multirow{3}{*}{$\begin{array}{l}\text { Eastern country } \\
\text { (Korea) }\end{array}$} & \multirow{3}{*}{$\begin{array}{l}\text { Cross-sectional } \\
\text { study }\end{array}$} & State anxiety, & $2.2(1.27-3.81)$ & \multirow[t]{3}{*}{ Marriage status } & \multirow[t]{3}{*}{$0.45(0.27-0.74)$} \\
\hline & & & Depression & $2.23(1.18-4.22)$ & & \\
\hline & & & Current smoking. & $2.28(1.47-3.55)$ & & \\
\hline \multirow{2}{*}{$\begin{array}{l}\text { Hideyuki Chiba, } \\
2012^{42}\end{array}$} & \multirow{2}{*}{$\begin{array}{l}\text { Eastern country } \\
\text { (Japan) }\end{array}$} & \multirow{2}{*}{$\begin{array}{l}\text { Cross-sectional } \\
\text { study }\end{array}$} & Alcohol & $1.398(1.040-1.880)$ & & \\
\hline & & & Smoking & $1.884(1.307-2.716)$ & & \\
\hline \multirow{2}{*}{$\begin{array}{l}\text { C. H. Park, } \\
2012^{38}\end{array}$} & \multirow{2}{*}{$\begin{array}{l}\text { Eastern country } \\
\text { (Korea) }\end{array}$} & \multirow{2}{*}{$\begin{array}{l}\text { Case control } \\
\text { study }\end{array}$} & Smoking & $2.827(1.932-4.664)$ & & \\
\hline & & & Coffee & $1.347(1.131-1.428)$ & & \\
\hline \multirow{3}{*}{$\begin{array}{l}\text { Naomi } \\
\text { Mochizuki, } \\
2018^{46}\end{array}$} & \multirow{3}{*}{$\begin{array}{l}\text { Eastern country } \\
\text { (Japan) }\end{array}$} & \multirow[t]{3}{*}{ Cohort study } & Current smoking & $1.34(1.12-1.61)$ & & \\
\hline & & & Alcohol consumption $20 \mathrm{~g} /$ day & $1.57(1.34-1.84)$ & & \\
\hline & & & High levels of stress & $1.4(1.17-1.68)$ & & \\
\hline \multirow{3}{*}{$\begin{array}{l}\text { Linda Sharp, } \\
2013^{10}\end{array}$} & \multirow{3}{*}{$\begin{array}{l}\text { Western country } \\
\text { (Ireland) }\end{array}$} & \multirow{3}{*}{$\begin{array}{l}\text { Case control } \\
\text { study }\end{array}$} & & & Folate & $0.34(0.18-0.64)$ \\
\hline & & & & & Vitamin B-6 & $0.30(0.16-0.55)$ \\
\hline & & & & & Vitamin B-2 & $0.35(0.19-0.66)$. \\
\hline $\begin{array}{l}\text { LESLEY } \\
\text { A. ANDERSON, } \\
2009^{11}\end{array}$ & $\begin{array}{l}\text { Western country } \\
\text { (Ireland) }\end{array}$ & $\begin{array}{l}\text { Case control } \\
\text { study }\end{array}$ & $\begin{array}{l}\text { Total alcohol consumption at } \\
\text { age } 21 \text { years }\end{array}$ & $2.24(1.35-3.74)$ & $\begin{array}{l}\text { Wine consumption at } 5 \\
\text { Years Prior to Interview }\end{array}$ & $0.45(0.27-0.75)$ \\
\hline $\begin{array}{l}\text { Helen } \\
\text { G. Mulholland, } \\
2009^{12}\end{array}$ & $\begin{array}{l}\text { Western country } \\
\text { (Ireland) }\end{array}$ & $\begin{array}{l}\text { Case control } \\
\text { study }\end{array}$ & Starch & $3.73(11.2-11.65)$ & & \\
\hline \multirow{4}{*}{$\begin{array}{l}\text { Mark } \\
\text { G. O'Doherty, } \\
2011^{13}\end{array}$} & \multirow{4}{*}{$\begin{array}{l}\text { Western country } \\
\text { (Ireland) }\end{array}$} & \multirow{4}{*}{$\begin{array}{l}\text { Case control } \\
\text { study }\end{array}$} & Total fat & $3.54(1.32-9.46)$ & & \\
\hline & & & Monounsaturated fat & $2.63(1.01-6.86)$ & & \\
\hline & & & Saturated fat & $2.79(1.11-7.04)$ & & \\
\hline & & & Processed meat & $4.67(|.7|-12.74)$ & & \\
\hline Qi Dai, $2016^{14}$ & $\begin{array}{l}\text { Western country } \\
\text { (Ireland) }\end{array}$ & $\begin{array}{l}\text { Case control } \\
\text { study }\end{array}$ & & & Magnesium & $0.12(0.02-0.73)$ \\
\hline $\begin{array}{l}\text { Seamus } \\
\text { J. Murphy, } 2010^{16}\end{array}$ & $\begin{array}{l}\text { Western country } \\
\text { (Ireland) }\end{array}$ & $\begin{array}{l}\text { Case control } \\
\text { study }\end{array}$ & & & Vitamin C & $0.46(0.24-0.90)$ \\
\hline \multirow{2}{*}{$\begin{array}{l}\text { Kun Wang, } \\
2019^{55}\end{array}$} & \multirow{2}{*}{$\begin{array}{l}\text { Eastern country } \\
\text { (China) }\end{array}$} & \multirow{2}{*}{$\begin{array}{l}\text { Cross-sectional } \\
\text { study }\end{array}$} & Ever smoking & $1.416(1.012-1.983)$ & & \\
\hline & & & $\begin{array}{l}\text { Frequent liquid food } \\
\text { consumption }\end{array}$ & $1.502(1.076-2.095)$ & & \\
\hline
\end{tabular}

(Continued) 
Table 4 (Continued).

\begin{tabular}{|c|c|c|c|c|c|c|}
\hline $\begin{array}{l}\text { First Author, } \\
\text { Publication } \\
\text { Year }\end{array}$ & Area (Country) & $\begin{array}{l}\text { Clinical Study } \\
\text { Design }\end{array}$ & Positive Related Factors & AOR $(95 \% \mathrm{Cl})$ & $\begin{array}{l}\text { Negative Related } \\
\text { Factors }\end{array}$ & AOR $(95 \% \mathrm{Cl})$ \\
\hline S. Peng, $2009^{60}$ & $\begin{array}{l}\text { Eastern country } \\
\text { (China) }\end{array}$ & $\begin{array}{l}\text { Cross-sectional } \\
\text { study }\end{array}$ & Alcohol & $3.22(1.92-5.39)$ & & \\
\hline Juan $\mathrm{Du}, 2007^{62}$ & $\begin{array}{l}\text { Eastern country } \\
\text { (China) }\end{array}$ & $\begin{array}{l}\text { Cross-sectional } \\
\text { study }\end{array}$ & Strong tea drinking & $1.62(1.18-2.23)$ & Divorced/widowed & $0.55(0.36-0.85)$ \\
\hline
\end{tabular}

Abbreviations: RE, reflux esophagitis; AOR, adjusted odds ratio.

position, the patients with upright reflux without esophagitis experienced reduced reflux in the supine position, postprandial reflux was increased in the patients without esophagitis, and bidirectional reflux with esophagitis gradually decreased after meals; the differences between the two reflux diseases in different positions and 24 $\mathrm{h}$ postprandial esophageal $\mathrm{pH}$ monitoring indicated that the pathological mechanisms of different types of gastroesophageal reflux diseases may be different, but postprandial habits and posture indeed affect reflux. ${ }^{88}$ Studies have shown that among patients with nocturnal acid reflux, changing the sleeping posture, ie, raising the head of the bed by $20 \mathrm{~cm}$, can effectively reduce the acid reflux time, acid clearance time, and number of reflux events $>5$ min. ${ }^{89}$ Another study showed that as a result of the use of a sleeppositioning device (SPD) among healthy subjects and the use of a left-side decubitus (SPD-L), the esophageal acid exposure time and reflux times were significantly lower than those associated with the right lateral position (SPD$\mathrm{R})$, any position with a standard wedge sleep aid device and the supine position. ${ }^{90}$

An interval of less than 2 hours between dinner and sleep, eating 2 hours before bed, and an interval of less than 3 hours between dinner and sleep were positively correlated with GERD. ${ }^{34,41,73}$ Studies have shown that when the interval between dinner and sleep is less than 2 hours, the percentage of reflux time is significantly higher than when the interval between dinner and sleep is greater than 2 hours $(22.6 \%$ vs $14.2 \% ; \mathrm{P}=0.012)$. There was no significant difference in the percentage of reflux time according to an interval between dinner and sleep less than 3 hours and an interval between dinner and sleep greater than 3 hours $(16.3 \%$ vs $14.6 \%, \mathrm{P}=0.798) .{ }^{91}$ This finding suggests that GERD patients should stop eating 3 hours before bedtime.

Smoking and alcohol consumption are positively correlated with GERD. This finding is consistent with the conclusions of previous studies. A meta-analysis found that smoking is a risk factor for $\mathrm{BE}$ and that alcohol consumption is a risk factor for GERD. ${ }^{92,93}$ However, wine is negatively correlated with RE 11 and $\mathrm{BE},{ }^{25}$ and beer is negatively correlated with GERD 23 and BE. ${ }^{17}$ Because the pathological changes associated with RE and $\mathrm{BE}$ are caused by the activation of inflammatory pathways by reflux substances, which leads to mucosal damage, ${ }^{94}$ we suspect that this finding may be related to the antioxidant substances in beer and wine..$^{95,96}$

Psychological factors were found to be positively correlated with GERD, NERD, and RE. However, it was found that the severity of anxiety was related to retrosternal pain and heartburn, while the levels of anxiety and depression were not associated with the number of reflux symptoms or the number of related reflux events reported by 24-hour $\mathrm{pH}$ impedance monitoring. ${ }^{97}$

Sleep time or quality is correlated with GERD. Previously, it was believed that a lack of sleep and GERD interact. Nocturnal acid reflux seriously affects the quality of sleep, and a lack of sleep can cause hyperalgesia of the esophageal mucosa to gastric acid. ${ }^{98}$ A recent study found that night awakening can induce nocturnal acid reactions. However, acid reflux at night does not cause awakening. ${ }^{99}$

We conclude that exercise is beneficial for GERD, but relevant research concerning the type and degree of exercise that are most suitable for reducing GERD is lacking. A study involving 10 healthy athletes found that running could cause a significant increase in the percentage of time with $\mathrm{PH}<4$ and that increasing the exercise intensity could lead to an increase in the frequency of reflux events and an extension of the duration of reflux. ${ }^{100}$

In our research, we found that lifestyle factors that increase abdominal pressure, such as belt tightness, ${ }^{74}$ wearing girdles or corsets, ${ }^{58}$ and constipation, ${ }^{44,64,70,75,78,79}$ are positively correlated with GERD and NERD. ${ }^{62}$ For example, 
Table 5 The Main Findings Related to the BE Across the Articles

\begin{tabular}{|c|c|c|c|c|c|c|}
\hline $\begin{array}{l}\text { First Author, } \\
\text { Publication } \\
\text { Year }\end{array}$ & Area (Country) & $\begin{array}{l}\text { Clinical Study } \\
\text { Design }\end{array}$ & $\begin{array}{l}\text { Positive Related } \\
\text { Factors }\end{array}$ & AOR $(95 \% \mathrm{Cl})$ & $\begin{array}{l}\text { Negative } \\
\text { Related } \\
\text { Factors }\end{array}$ & AOR $(95 \% \mathrm{Cl})$ \\
\hline \multirow{2}{*}{$\begin{array}{l}\text { Olivia } \\
\text { M Thompson, } \\
2009^{24}\end{array}$} & \multirow{2}{*}{$\begin{array}{l}\text { Western } \\
\text { country(American) }\end{array}$} & \multirow{2}{*}{$\begin{array}{l}\text { Case control } \\
\text { study }\end{array}$} & & & Vegetables & $0.33(0.17-0.63)$ \\
\hline & & & & & $\begin{array}{l}\text { Vegetables and } \\
\text { fruit }\end{array}$ & $0.39(0.2 \mathrm{I}-0.75)$ \\
\hline $\begin{array}{l}\text { Ai Kubo, } \\
2004^{25}\end{array}$ & $\begin{array}{l}\text { Western } \\
\text { country(American) }\end{array}$ & $\begin{array}{l}\text { Case control } \\
\text { study }\end{array}$ & & & Wine drinkers & $0.44(0.2-0.99)$ \\
\hline \multirow[t]{7}{*}{ Li Jiao, $2013^{26}$} & \multirow[t]{7}{*}{$\begin{array}{l}\text { Western } \\
\text { country(American) }\end{array}$} & \multirow[t]{7}{*}{$\begin{array}{l}\text { Case control } \\
\text { study }\end{array}$} & & & $\begin{array}{l}\text { Dark green } \\
\text { vegetables }\end{array}$ & $0.46(0.26-0.8 \mathrm{I})$ \\
\hline & & & & & Legumes & $0.52(0.30-0.90)$ \\
\hline & & & & & Total fiber & $0.50(0.28-0.90)$ \\
\hline & & & & & Isoflavones & $0.45(0.25-0.81)$ \\
\hline & & & & & Total folate & $0.52(0.30-0.67)$ \\
\hline & & & & & Vitamin E & $0.46(0.26-0.83)$ \\
\hline & & & & & Lutein & $0.45(0.26-0.79)$ \\
\hline $\begin{array}{l}\text { Jessica } \\
\text { L. Petrick, } \\
2015^{27}\end{array}$ & $\begin{array}{l}\text { Western } \\
\text { country(American) }\end{array}$ & $\begin{array}{l}\text { Case control } \\
\text { study }\end{array}$ & & & Anthocyanidins & $0.49(0.30-0.80)$ \\
\hline $\begin{array}{l}\text { Douglas } \\
\text { A. Corley, } \\
2008^{29}\end{array}$ & $\begin{array}{l}\text { Western } \\
\text { country(American) }\end{array}$ & $\begin{array}{l}\text { Case control } \\
\text { study }\end{array}$ & & & $\begin{array}{l}\text { Dietary iron } \\
\text { intakes }\end{array}$ & $\begin{array}{l}0.37 \\
(0.17-0.80)\end{array}$ \\
\hline $\begin{array}{l}\text { Stephen Lam, } \\
2017^{33}\end{array}$ & $\begin{array}{l}\text { Western } \\
\text { country(UK) }\end{array}$ & Cohort study & & & $\begin{array}{l}\text { Standing } \\
\text { occupation }\end{array}$ & $0.5 \mathrm{I}(0.3 \mathrm{I}-0.83)$ \\
\hline \multirow{2}{*}{$\begin{array}{l}\text { Linda Sharp, } \\
2013^{10}\end{array}$} & \multirow{2}{*}{$\begin{array}{l}\text { Western country } \\
\text { (Ireland) }\end{array}$} & \multirow{2}{*}{$\begin{array}{l}\text { Case control } \\
\text { study }\end{array}$} & \multirow[t]{2}{*}{ Vitamin B-12 } & \multirow[t]{2}{*}{$2.11(1.12-3.98)$} & Folate & $0.40(0.21-0.75)$ \\
\hline & & & & & Vitamin B-6 & $0.31(0.16-0.58)$ \\
\hline $\begin{array}{l}\text { Helen } \\
\text { G. Mulholland, } \\
2009^{12}\end{array}$ & $\begin{array}{l}\text { Western country } \\
\text { (Ireland) }\end{array}$ & $\begin{array}{l}\text { Case control } \\
\text { study }\end{array}$ & & & Fiber & $0.40(0.22-0.73)$ \\
\hline Qi Dai, $2016^{14}$ & $\begin{array}{l}\text { Western country } \\
\text { (Ireland) }\end{array}$ & $\begin{array}{l}\text { Case control } \\
\text { study }\end{array}$ & & & Magnesium & $0.24(0.06-0.96)$ \\
\hline $\begin{array}{l}\text { Nitin Shivappa, } \\
2017^{15}\end{array}$ & $\begin{array}{l}\text { Western country } \\
\text { (Ireland) }\end{array}$ & $\begin{array}{l}\text { Case control } \\
\text { study }\end{array}$ & $\begin{array}{l}\text { Dietary inflammatory } \\
\text { index }\end{array}$ & $2.05(1.22-3.47)$ & & \\
\hline $\begin{array}{l}\text { RA Filiberti, } \\
2017^{31}\end{array}$ & $\begin{array}{l}\text { Western country } \\
\text { (Italy) }\end{array}$ & $\begin{array}{l}\text { Case control } \\
\text { study }\end{array}$ & $\begin{array}{l}\text { Former coffee drinkers } \\
\text { higher with duration cup } \\
\text { per day }>\text { I }\end{array}$ & $3.79(1.31-11.0)$ & & \\
\hline $\begin{array}{l}\text { Aaron P. Thrift, } \\
2011^{17}\end{array}$ & $\begin{array}{l}\text { Western country } \\
\text { (Australia) }\end{array}$ & $\begin{array}{l}\text { Case control } \\
\text { study }\end{array}$ & & & Beer & $0.49(0.25-0.96)$ \\
\hline $\begin{array}{l}\text { Torukiri } \\
\text { I. Ibiebele, } \\
2013^{18}\end{array}$ & $\begin{array}{l}\text { Western country } \\
\text { (Australia) }\end{array}$ & $\begin{array}{l}\text { Case control } \\
\text { study }\end{array}$ & Fruits & $1.83(1.02-3.29)$ & $\begin{array}{l}\text { Total } \beta- \\
\text { carotene }\end{array}$ & $0.45(0.20-1.00)$ \\
\hline
\end{tabular}

(Continued) 
Table 5 (Continued).

\begin{tabular}{|c|c|c|c|c|c|c|}
\hline $\begin{array}{l}\text { First Author, } \\
\text { Publication } \\
\text { Year }\end{array}$ & Area (Country) & $\begin{array}{l}\text { Clinical Study } \\
\text { Design }\end{array}$ & $\begin{array}{l}\text { Positive Related } \\
\text { Factors }\end{array}$ & AOR (95\% Cl) & $\begin{array}{l}\text { Negative } \\
\text { Related } \\
\text { Factors }\end{array}$ & AOR $(95 \% \mathrm{CI})$ \\
\hline $\begin{array}{l}\text { Jessie Steevens, } \\
2010^{21}\end{array}$ & $\begin{array}{l}\text { Western country } \\
\text { (Netherland) }\end{array}$ & Cohort study & $\begin{array}{l}\text { Former cigarette } \\
\text { smokers }\end{array}$ & $1.33(1.00-1.77)$ & & \\
\hline $\begin{array}{l}\text { Yan-Hua Chen, } \\
2019^{59}\end{array}$ & $\begin{array}{l}\text { Eastern } \\
\text { country(China) }\end{array}$ & $\begin{array}{l}\text { Cross-sectional } \\
\text { study }\end{array}$ & Tea & $1.695(1.043-2.754)$ & & \\
\hline S. Peng, $2009^{60}$ & $\begin{array}{l}\text { Eastern } \\
\text { country(China) }\end{array}$ & $\begin{array}{l}\text { Cross-sectional } \\
\text { study }\end{array}$ & Alcohol consumption & $2.67(1.09-6.56)$ & & \\
\hline $\begin{array}{l}\text { YIN CaiQiao, } \\
2016^{67}\end{array}$ & $\begin{array}{l}\text { Eastern } \\
\text { country(China) }\end{array}$ & $\begin{array}{l}\text { Cross-sectional } \\
\text { study }\end{array}$ & High fat high & $2.216(1.06-2.695)$ & & \\
\hline $\begin{array}{l}\text { P. J. Veugelers, } \\
2006^{22}\end{array}$ & $\begin{array}{l}\text { Western country } \\
\text { (Canada) }\end{array}$ & $\begin{array}{l}\text { Case-control } \\
\text { study }\end{array}$ & Liquor & $3.06(1.23-7.62)$ & Vitamin C & $0.44(0.2-0.98)$ \\
\hline
\end{tabular}

Abbreviations: BE, Barrett's esophagus; AOR, adjusted odds ratio.

in obese people, the factors related to GERD include an increase in the diaphragm pressure gradient caused by an increase in abdominal pressure caused by central obesity rather than body mass index (BMI). ${ }^{101}$

We also noticed that some occupational characteristics are related to GERD. Standing occupational activity ${ }^{33}$ was negatively correlated with BE. Sedentary labor, ${ }^{49}$ physical labor, ${ }^{77}$ and physical labor intensity ${ }^{79}$ are positively correlated with GERD. Marital status, educational experience, and the living environment (urban or rural) are not associated with GERD, but it has been found that altitude and length of residence in the same area ${ }^{54}$ are positively correlated with GERD, which may be the result of bias caused by the research design.

Studies ${ }^{102}$ have shown that health-related behaviors do not occur simultaneously in individuals but rather in clusters. The investigation of four health-related behaviors, ie, smoking, drinking, diet and sports activities, showed that the aggregation of different behaviors has sex differences. In addition, these factors are related to age. Additionally, the behavioral risk factors for noncommunicable diseases ${ }^{103}$ differ among groups with different socioeconomic statuses, and their dietary and lifestyle habits differ. In low-income and low-middle-income countries, groups with a low socioeconomic status more commonly smoke and consume alcohol than those with a high socioeconomic status. Compared with people with a high socioeconomic status, those with a low socioeconomic status consume less fruits, vegetables, fish and fiber; compared with people with a low socioeconomic status, people with a high socioeconomic status exercise less and consume more fat, salt, and processed food. These findings further confirm that dietary structure ${ }^{104}$ differs by age, gender, socioeconomic status, region (urban/rural) and residential area. In our research, we found that among all dietary and lifestyle risk factors, those associated with GERD in Eastern countries are eating habits, postprandial behaviors, milk, food taste, energy, time from dinner to sleep, psychological status, marital status, sleep, living environment, etc. The relevant influencing factors only identified in Western countries are staple food, fiber, vitamins, trace elements, dietary inflammatory factors, and soft drinks. However, whether differences exist in the incidence of GERD according to region, sex and age based on regional differences in diet and lifestyle habits requires further research and confirmation in follow-up studies.

Some studies have involved subgroup analyses based on symptoms, endoscopy, and pathological examinations. For example, two articles ${ }^{35,37}$ simultaneously studied NERD and RE, four articles ${ }^{10,12,14,60}$ conducted research concerning $\mathrm{RE}$ and $\mathrm{BE}$, one article ${ }^{22}$ conducted research concerning GERD and BE, and one article ${ }^{62}$ conducted research concerning GERD, NERD, and RE simultaneously, further indicating that differences in diet and lifestyle exist among GERD, NERD, RE and BE. Since the risk factors for different types of gastroesophageal reflux differ, future research should focus on the different types of risk factors, targeted prevention and control to prevent the occurrence of these diseases. 
There are some limitations in our study. We only searched two English databases and two Chinese databases, which may only reflect GERD-related factors associated with diet and lifestyle to a certain extent. Due to the heterogeneity among the studies and the different definitions and measurement methods used for each factor, we cannot make quantitative inferences regarding each variable or determine a dose effect. Additionally, the classification and division of various dietary factors cannot be absolutely accurate. For example, regarding sweets, the sweet taste is mainly due to the content of starch, and sweets might not be categorized as fat-related fried foods or greasy foods despite their fat content. Furthermore, we identified the independent factors influencing only GERD in the current literature. It is well known that a single influencing factor does not cause the disease. The disease is usually the result of multiple factors. What type of interaction exists among these factors? Further research is needed.

\section{Conclusion}

GERD is a chronic digestive system disease caused by multiple factors and multiple pathways. We found that various dietary and lifestyle factors affect the occurrence of GERD. Among GERD, RE, NERD, and BE, the associated diet and lifestyle factors are different. There were also differences in the diet- and lifestyle-related factors related to GERD according to region. Whether such differences are the cause of the differences in incidence requires further research. Whether the differences in diet and lifestyle structure associated with age and sex are also reasons for the difference in incidence needs further study.

\section{Author Contributions}

All authors made a significant contribution to the work reported, as related to the conception, study design, execution, acquisition of data, analysis and interpretation, or all of the above; took part in drafting, revising or critically reviewing the article; gave final approval of the version to be published; have agreed on the journal to which the article has been submitted; and agree to be accountable for all aspects of the work.

\section{Funding}

This work was supported by the National Natural Science Foundation of China (No. 81774450) and Science and Technology Planning Project of Guangdong Province, China (No. 2017A020215107).

\section{Disclosure}

The authors have no conflicts of interest to report in this work.

\section{References}

1. Vakil N, van Zanten SV, Kahrilas P, Dent J, Jones R. The Montreal definition and classification of gastroesophageal reflux disease: a global evidence-based consensus. Am J Gastroenterol. 2006;101(8):1900-1920. doi:10.1111/j.1572-0241.2006.00630.x

2. Chen J, Brady P. Gastroesophageal Reflux Disease: pathophysiology, Diagnosis, and Treatment. Gastroenterol Nurs. 2019;42 (1):20-28. doi:10.1097/SGA.0000000000000359

3. Eusebi LH, Ratnakumaran R, Yuan Y, Solaymani-Dodaran M, Bazzoli F, Ford AC. Global prevalence of, and risk factors for, gastro-oesophageal reflux symptoms: a meta-analysis. Gut. 2018;67(3):430-440. doi:10.1136/gutjnl-2016-313589

4. Richter JE, Rubenstein JH. Presentation and Epidemiology of Gastroesophageal Reflux Disease. Gastroenterology. 2018;154 (2):267-276. doi:10.1053/j.gastro.2017.07.045

5. Freedberg DE, Kim LS, Yang YX, Risks T. and Benefits of Long-term Use of Proton Pump Inhibitors: expert Review and Best Practice Advice From the American Gastroenterological Association. Gastroenterology. 2017;152(4):706-715. doi:10.1053/ j.gastro.2017.01.031

6. Sethi S, Richter JE. Diet and gastroesophageal reflux disease: role in pathogenesis and management. Curr Opin Gastroenterol. 2017;33(2):107-111. doi:10.1097/MOG.0000000000000337

7. Patti MG. An evidence-based approach to the treatment of gastroesophageal reflux disease. JAMA Surg. 2016;151(1):73-78. doi:10.1001/jamasurg.2015.4233

8. Mone I, Kraja B, Bregu A, et al. Adherence to a predominantly Mediterranean diet decreases the risk of gastroesophageal reflux disease: a cross-sectional study in a South Eastern European population. Dis Esophagus. 2016;29(7):794-800. doi:10.1111/dote.12384

9. Çela L, Kraja B, Hoti K, et al. Lifestyle characteristics and gastroesophageal reflux disease: a population-based study in Albania. Gastroent Res Pract. 2013;2013.

10. Sharp L, Carsin AE, Cantwell MM, Anderson LA, Murray LJ. Intakes of dietary folate and other B vitamins are associated with risks of esophageal adenocarcinoma, Barrett's esophagus, and reflux esophagitis. J Nutr. 2013;143(12):1966-1973. doi:10.3945/jn.113.174664

11. Anderson LA, Cantwell MM, Watson RGP, et al. The association between alcohol and reflux esophagitis, barrett's esophagus, and esophageal adenocarcinoma. Gastroenterology. 2009;136 (3):799-805. doi:10.1053/j.gastro.2008.12.005

12. Mulholland HG, Cantwell MM, Anderson LA, et al. Glycemic index, carbohydrate and fiber intakes and risk of reflux esophagitis, Barrett's esophagus, and esophageal adenocarcinoma. Cancer Causes Control. 2009;20(3):279-288. doi:10.1007/s10552-008-9242-6

13. O’Doherty MG, Cantwell MM, Murray LJ, Anderson LA, Abnet CC. Dietary fat and meat intakes and risk of reflux esophagitis, Barrett's esophagus and esophageal adenocarcinoma. Int $J$ Cancer. 2011;129(6):1493-1502. doi:10.1002/ijc.26108

14. Dai Q, Cantwell MM, Murray LJ, Zheng W, Anderson LA, Coleman HG. Dietary magnesium, calcium:magnesium ratio and risk of reflux oesophagitis, Barrett's oesophagus and oesophageal adenocarcinoma: a population-based case-control study. $\mathrm{Br}$ J Nutr. 2016;115(2):342-350. doi:10.1017/S0007114515004444

15. Shivappa N, Hebert JR, Anderson LA, et al. Dietary inflammatory index and risk of reflux oesophagitis, Barrett's oesophagus and oesophageal adenocarcinoma: a population-based case-control study. $\quad B r \quad J \quad$ Nutr. 2017;117(9):1323-1331. doi:10.1017/ S0007114517001131 
16. Murphy SJ, Anderson LA, Ferguson HR, et al. Dietary antioxidant and mineral intake in humans is associated with reduced risk of esophageal adenocarcinoma but not reflux esophagitis or Barrett's esophagus. $J \quad$ Nutr. 2010;140(10):1757-1763. doi:10.3945/jn.110.124362

17. Thrift AP, Pandeya N, Smith KJ, et al. Lifetime alcohol consumption and risk of barrett's esophagus. Am J Gastroenterol. 2011;106(7):1220-1230. doi:10.1038/ajg.2011.89

18. Ibiebele TI, Hughes MC, Nagle CM, Bain CJ, Whiteman DC, Webb PM. Dietary antioxidants and risk of Barrett's esophagus and adenocarcinoma of the esophagus in an Australian population. Int J Cancer. 2013;133(1):214-224. doi:10.1002/ijc.28016

19. Boguradzka A, Tarnowski W, Cabaj H. Gastroesophageal reflux in alcohol-abusing patients. Pol Arch Med Wewn. 2011;121 (7-8):230-236.

20. Jarosz M, Taraszewska A. Risk factors for gastroesophageal reflux disease: the role of diet. Przeglad Gastroenterologiczny. 2014;9(5):297-301. doi:10.5114/pg.2014.46166

21. Steevens J, Schouten LJ, Driessen AL, et al. A prospective cohort study on overweight, smoking, alcohol consumption, and risk of Barrett's esophagus. Cancer Epidemiol Biomarkers Prev. 2011;20 (2):345-358. doi:10.1158/1055-9965.EPI-10-0636

22. Veugelers PJ, Porter GA, Guernsey DL, Casson AG. Obesity and lifestyle risk factors for gastroesophageal reflux disease, Barrett esophagus and esophageal adenocarcinoma. Dis Esophagus. 2006;19(5):321-328. doi:10.1111/j.1442-2050.2006.00602.x

23. Kubo A, Block G, Quesenberry CJ, Buffler P, Corley DA. Dietary guideline adherence for gastroesophageal reflux disease. Bmc Gastroenterol. 2014;14:144. doi:10.1186/1471-230X-14-144

24. Thompson OM, Beresford SA, Kirk EA, Vaughan TL. Vegetable and fruit intakes and risk of Barrett's esophagus in men and women. Am J Clin Nutr. 2009;89(3):890-896. doi:10.3945/ajcn.2008.26497

25. Kubo A, Levin TR, Block G, et al. Alcohol types and sociodemographic characteristics as risk factors for Barrett'sesophagus. Gastroenterology. 2009;136(3):806-815. doi:10.1053/j.gastro.200 8.11.042

26. Jiao L, Kramer JR, Rugge M, et al. Dietary intake of vegetables, folate, and antioxidants and the risk of Barrett's esophagus. Cancer Causes Control. 2013;24(5):1005-1014. doi:10.1007/ s10552-013-0175-3

27. Petrick JL, Steck SE, Bradshaw PT, et al. Dietary flavonoid intake and Barrett's esophagus in western Washington State. Ann Epidemiol. 2015;25(10):730-735. doi:10.1016/j.annepidem.20 15.05.010

28. Friedenberg FK, Rai J, Vanar V, et al. Prevalence and risk factors for gastroesophageal reflux disease in an impoverished minority population. Obes Res Clin Pract. 2010;4(4):e261-e269. doi:10.1016/j.orcp.2010.06.001

29. Corley DA, Kubo A, Levin TR, et al. Iron intake and body iron stores as risk factors for Barrett's esophagus: a community-based study. Am J Gastroenterol. 2008;103(12):2997-3004. doi:10.111 1/j.1572-0241.2008.02156.x

30. Nilsson M, Johnsen R, Ye W, Hveem K, Lagergren J. Lifestyle related risk factors in the aetiology of gastro-oesophageal reflux. Gut. 2004;53(12):1730-1735. doi:10.1136/gut.2004.043265

31. Filiberti RA, Fontana V, De Ceglie A, et al. Association between coffee or tea drinking and Barrett's esophagus or esophagitis: an Italian study. Eur J Clin Nutr. 2017;71(8):980-986. doi:10.1038/ ejcn.2017.64

32. Dore MP, Maragkoudakis E, Fraley K, et al. Diet, lifestyle and gender in gastro-esophageal reflux disease. Dig Dis Sci. 2008;53 (8):2027-2032. doi:10.1007/s10620-007-0108-7

33. Lam S, Alexandre L, Luben R, Hart AR. The association between physical activity and the risk of symptomatic Barrett's oesophagus: a UK prospective cohort study. Eur J Gastroenterol Hepatol. 2018;30(1):71-75. doi:10.1097/MEG.0000000000000998
34. Ahmed S, Jamil S, Shaikh H, Abbasi M. Effects of life style factors on the symptoms of gastro esophageal reflux disease: a cross sectional study in a Pakistani population. Pak J Med Sci. 2020;36(2):115-120. doi:10.12669/pjms.36.2.1371

35. Nam SY, Park BJ, Cho YA, et al. Different effects of dietary factors on reflux esophagitis and non-erosive reflux disease in 11,690 Korean subjects. J Gastroenterol. 2017;52(7):818-829. doi:10.1007/s00535-016-1282-1

36. Ju G, Yoon IY, Lee SD, Kim N. Relationships between sleep disturbances and gastroesophageal reflux disease in Asian sleep clinic referrals. J Psychosom Res. 2013;75(6):551-555. doi:10.1016/j.jpsychores.2013.10.004

37. Choi JM, Yang JI, Kang SJ, et al. Association between anxiety and depression and gastroesophageal reflux disease: results from a large cross-sectional study. J Neurogastroenterol. 2018;24 (4):593-602. doi:10.5056/jnm18069

38. Park $\mathrm{CH}$, Kim KO, Baek IH, et al. Differences in the risk factors of reflux esophagitis according to age in Korea. Dis Esophagus. 2014;27(2):116-121. doi:10.1111/j.1442-2050.2012.01417.x

39. Rosaida MS, Goh KL. Gastro-oesophageal reflux disease, reflux oesophagitis and non-erosive reflux disease in a multiracial Asian population: a prospective, endoscopy based study. Eur J Gastroenterol Hepatol. 2004;16(5):495-501. doi:10.1097/ 00042737-200405000-00010

40. Shaha M, Perveen I, Alamgir MJ, Masud MH, Rahman MH. Prevalence and risk factors for gastro-esophageal reflux disease in the North-Eastern part of Bangladesh. Bangladesh Med Res Counc Bull. 2012;38(3):108-113. doi:10.3329/bmrcb.v38i 3.14338

41. Fujiwara Y, MacHida A, Watanabe Y, et al. Association between dinner-to-bed time and gastro-esophageal reflux disease. Am $J$ Gastroenterol. 2005;100(12):2633-2636. doi:10.1111/j.15720241.2005.00354.x

42. Chiba H, Gunji T, Sato H, et al. A cross-sectional study on the risk factors for erosive esophagitis in young adults. Intern Med. 2012;51(11):1293-1299. doi:10.2169/internalmedicine.51.7241

43. Murao T, Sakurai K, Mihara S, Marubayashi T, Murakami Y, Sasaki Y. Lifestyle change influences on GERD in Japan: a study of participants in a health examination program. Dig Dis Sci. 2011;56(10):2857-2864. doi:10.1007/s10620-011-1679-x

44. Miyamoto M, Haruma K, Kuwabara M, Nagano M, Okamoto T, Tanaka M. High incidence of newly-developed gastroesophageal reflux disease in the Japanese community: a 6-year follow-up study. J Gastroenterol Hepatology. 2008;23(3):393-397. doi:10.1111/j.1440-1746.2007.05043.x

45. Matsuki N, Fujita T, Watanabe N, et al. Lifestyle factors associated with gastroesophageal reflux disease in the Japanese population. J Gastroenterol. 2013;48(3):340-349. doi:10.1007/ s00535-012-0649-1

46. Mochizuki N, Fujita T, Kobayashi M, et al. Factors associated with the presentation of erosive esophagitis symptoms in health checkup subjects: a prospective, multicenter cohort study. PLoS One. 2018;13(5):e196848. doi:10.1371/journal.pone.0196848

47. Awadalla NJ, Al-Musa HM. Insomnia among primary care adult population in Aseer region of Saudi Arabia: gastroesophageal reflux disease and body mass index correlates. Biol Rhythm Res. 2019;1-11. doi:10.1080/09291016.2019.1656933

48. Eslami O, Shahraki M, Bahari A, Shahraki T. Dietary habits and obesity indices in patients with gastro-esophageal reflux disease: a comparative cross-sectional study. Bmc Gastroenterol. 2017;17 (1):132. doi:10.1186/s12876-017-0699-1

49. Kumar S, Sharma S, Norboo T, et al. Population based study to assess prevalence and risk factors of gastroesophageal reflux disease in a high altitude area. Indian J Gastroenterol. 2011;30 (3):135-143. doi:10.1007/s12664-010-0066-4 
50. Chowdhury SD, George G, Ramakrishna K, et al. Prevalence and factors associated with gastroesophageal reflux disease in southern India: a community-based study. Indian $J$ Gastroenterol. 2019;38(1):77-82. doi:10.1007/s12664-018-00931-6

51. Wang HY, Leena KB, Plymoth A, et al. Prevalence of gastro-esophageal reflux disease and its risk factors in a community-based population in southern India. Bmc Gastroenterol. 2016;16(1). doi:10.1186/s12876-016-0452-1

52. Sharma PK, Ahuja V, Madan K, Gupta S, Raizada A, Sharma MP. Prevalence, severity, and risk factors of symptomatic gastroesophageal reflux disease among employees of a large hospital in Northern India. Indian J Gastroenterology. 2011;30(3):128-134. doi:10.1007/s12664-010-0065-5

53. Bhatia SJ, Reddy DN, Ghoshal UC, et al. Epidemiology and symptom profile of gastroesophageal reflux in the Indian population: report of the Indian Society of Gastroenterology Task Force. Indian J Gastroenterol. 2011;30(3):118-127. doi:10.1007/ s12664-011-0112-x

54. Zhang H, Gao W, Wang L, et al. A population-based study on prevalence and risk factors of gastroesophageal reflux disease in the Tibet Autonomous Region, China. PeerJ. 2019;2019(2):154.

55. Wang K, Zhang L, He ZH, et al. A population-based survey of gastroesophageal reflux disease in a region with high prevalence of esophageal cancer in China. Chin Med J. 2019;132 (13):1516-1523. doi:10.1097/CM9.0000000000000275

56. He J, Ma X, Zhao Y, et al. A population-based survey of the epidemiology of symptom-defined gastroesophageal reflux disease: the Systematic Investigation of Gastrointestinal Diseases in China. BMC Gastroenterol. 2010;10:10. doi:10.1186/1471230X-10-94

57. Gong Y, Zeng Q, Yan Y, Han C, Zheng Y. Association between lifestyle and gastroesophageal reflux disease questionnaire scores: a cross-sectional study of 37442 chinese adults. Gastroent Res Pract. 2019;2019.

58. Yuan LZ, Yi P, Wang GS, et al. Lifestyle intervention for gastroesophageal reflux disease: a national multicenter survey of lifestyle factor effects on gastroesophageal reflux disease in China. Ther Adv Gastroenterol. 2019;12.

59. Chen YH, Yu HC, Lin KH, Lin HS, Hsu PI. Prevalence and risk factors for Barrett's esophagus in Taiwan. World J Gastroenterol. 2019;25(25):3231-3241. doi:10.3748/wjg.v25.i25.3231

60. Peng S, Cui Y, Xiao YL, et al. Prevalence of erosive esophagitis and Barretts esophagus in the adult Chinese population. Endoscopy. 2009;41(12):1011-1017. doi:10.1055/s-00291215291

61. Ma XQ, Cao Y, Wang R, et al. Prevalence of, and factors associated with, gastroesophageal reflux disease: a population-based study in Shanghai, China. Dis Esophagus. 2009;22(4):317-322. doi:10.1111/j.1442-2050.2008.00904.x

62. Du J, Liu J, Zhang H, Yu CH, Li YM. Risk factors for gastroesophageal reflux disease, reflux esophagitis and non-erosive reflux disease among Chinese patients undergoing upper gastrointestinal endoscopic examination. World $J$ Gastroenterol. 2007;13(45):6009-6015. doi:10.3748/wjg.v13.45.6009

63. Zhang M, Bin W, Chun-fang Z. Investigation on the prevalence and influencing factors of gastroesophageal reflux disease in Shiyan City. Hainan Me J. 2018;29(20):2917-2920.

64. Xiao-jun Y, Chen W, Chuan-bin C, et al. Epidemiologic investigation and analysis of gastroesophageal reflux disease in the population of Golmud in Qinghai Province. Chine J General Pract. 2018;16(8):1386-1388.

65. Yi W, Shengliang Z. Related risk factors of non-erosive reflux disease. Int J Digestive Dis. 2018;38(1):32-36.

66. Chenglin J, Qizhi C, Xiujin Z, Liusheng Y, Hualiang R. Epidemiologic Study on Gastroesophageal Reflux Disease in She Population. Chin J Gastroenterol. 2016;1(3):179-182.
67. Yin C, Zhang J, Wang J. Investigation on the relationship between eating habits and Barrett's esophagus. Shaanxi Med J. 2016;1 (3):368-370.

68. Rong L, Li K, Sen-Yuan Z, Hui-Min L, Hou LH. Symptomatic gastroesophageal reflux disease among Uygur residents in Urumqi: an epidemiological study and preventive treatment. World Chin J Digestology. 2013;33:3715-3719. doi:10.11569/ wcjd.v21.i33.3715

69. Chu J, Yan-hui S, Xue-ying Q, et al. A community-based epidemiologic study on gastroesophageal reflux disease in Haidian district of Beijing. Chin J Prevent Med. 2010;44(6):516-521.

70. Xu-de S, Wen W, Hui-jun Z. Epidemiologic survey on gastroesophageal reflux disease in the population of Fujian province. Chin J Digestion. 2010;1(6):386-390.

71. Liping $\mathrm{C}$, Zaiwei H, Bing X. Risk factors and clinical characteristics of gastroesophageal reflux disease: analysis based on a prospective database of functional gastrointestinal disease. J Southern Med Univ. 2016;36(05):710-713.

72. Hui-xin C, Li-shou $\mathrm{X}$, An-gao $\mathrm{X}$, et al. The risk factors and impact of gastroesophageal reflux disease on quality of life in general population. Chin J Internal Med. 2006;1(3):202-205.

73. Xiaodan L, Zhuoqin J, Niandi T. Correlation between lifestyle and dietary habit and gastroesophageal reflux disease. New Med. 2018;49(1):52-56.

74. Lingzhi Y, Dan T, Jin P, Nanfang Q, Chun Y, Fen W. Study on lifestyle in patients with gastroesophageal reflux disease. J Central South Univ Med Sci. 2017;42(5):558-564.

75. Hongliang G, Wentao F, Ping Y. Analysis of Risk Factors for Gastroesophageal Reflux Disease. Chin J Gastroenterol. 2012;17 (8):483-487.

76. Yanwei Y, Qianqian S, Bin R. Analysis of risk factors of gastroesophageal reflux disease in 303 patients. Chongqing Med. 2012;41(18):1838-1840.

77. Xuan J, Shujie W, Suqiao Z, et al. Analysis of the risk factors of gastrointesital esophageal reflux disease and its clinical features. Chine J Pract Int Med. 2011;31(10):774-776.

78. Hu S, Zhang M, Niu X. Zhou Epidemiological investigation of gastroesophageal reflux disease in outpatients. J Pract Med. 2009;25(1):141-143.

79. Hong Z, Jing X, You-ming L, et al. Epidemiologic study on symptomatic gastroesophageal reflux disease and its risk factors in outpatient populations of internal medicine in Zhejiang province. Chine J Digestion. 2007;27(07):443-446.

80. Lopez-Colombo A, Pacio-Quiterio MS, Jesus-Mejenes LY, et al. Risk factors associated with gastroesophageal reflux disease relapse in primary care patients successfully treated with a proton pump inhibitor. Rev Gastroenterol Mex. 2017;82 (2):106-114. doi:10.1016/j.rgmx.2016.09.001

81. Gomes DC, Dantas RO. Acidic and neutral liquid ingestion in patients with gastroesophageal reflux disease. Arq Gastroenterol. 2014;51(3):217-220. doi:10.1590/S0004-28032014000300010

82. Agrawal A, Tutuian R, Hila A, Freeman J, Castell DO. Ingestion of acidic foods mimics gastroesophageal reflux during $\mathrm{pH}$ monitoring. Dig Dis Sci. 2005;50(10):1916-1920. doi:10.1007/ s10620-005-2961-6

83. Watanabe F, Bito T. Vitamin B12 sources and microbial interaction. Exp Biol Med. 2018;243(2):148-158. doi:10.1177/ 1535370217746612

84. Surdea-Blaga T, Negrutiu DE, Palage M, Dumitrascu DL. Food and Gastroesophageal Reflux Disease. Curr Med Chem. 2019;26 (19):3497-3511. doi:10.2174/0929867324666170515123807

85. Kiraly A, Suto G, Czimmer J, Horvath OP, Mozsik G. Failure of capsaicin-containing red pepper sauce suspension to induce esophageal motility response in patients with Barrett's esophagus. $J$ Physiol Paris. 2001;95(1-6):197-200. doi:10.1016/S09284257(01)00025-0 
86. Milke P, Diaz A, Valdovinos MA, Moran S. Gastroesophageal reflux in healthy subjects induced by two different species of chilli (Capsicum annum). Dig Dis. 2006;24(1-2):184-188. doi: $10.1159 / 000090323$

87. Hila A, Bouali H, Xue S, Knuff D, Castell DO. Postprandial stomach contents have multiple acid layers. $J$ Clin Gastroenterol. 2006;40(7):612-617. doi:10.1097/00004836200608000-00010

88. Shay SS, Johnson LF. Upright refluxers without esophagitis differentiated from bipositional refluxers with esophagitis by simultaneous manometry and $\mathrm{pH}$ monitoring conducted in two postures before and after a meal. Am J Gastroenterol. 1994;89 (7):992-1002.

89. Khan BA, Sodhi JS, Zargar SA, et al. Effect of bed head elevation during sleep in symptomatic patients of nocturnal gastroesophageal reflux. J Gastroenterol Hepatol. 2012;27(6):1078-1082. doi:10.1111/j.1440-1746.2011.06968.x

90. Person E, Rife C, Freeman J, Clark A, Castell DO, Novel Sleep A. Positioning Device Reduces Gastroesophageal Reflux: a Randomized Controlled Trial. J Clin Gastroenterol. 2015;49 (8):655-659. doi:10.1097/MCG.0000000000000359

91. King AL, Baburajan B, Wong T, Anggiansah R, Anggiansah A. Determinants of Abnormal Supine Reflux in 24-Hour $\mathrm{pH}$ Recordings. Dig Dis Sci. 2007;52(10):2844-2849. doi:10.1007/ s10620-006-9707-y

92. Andrici J, Cox MR, Eslick GD. Cigarette smoking and the risk of Barrett's esophagus: a systematic review and meta-analysis. J Gastroenterol Hepatol. 2013;28(8):1258-1273. doi:10.1111/ jgh. 12230

93. Pan J, Cen L, Chen W, Yu C, Li Y, Shen Z. Alcohol consumption and the risk of gastroesophageal reflux disease: a systematic review and meta-analysis. Alcohol Alcohol. 2019;54(1):62-69. doi:10.1093/alcalc/agy063

94. Souza RF. Reflux esophagitis and its role in the pathogenesis of Barrett's metaplasia. J Gastroenterol. 2017;52(7):767-776. doi:10.1007/s00535-017-1342-1

95. Martinez-Gomez A, Caballero I, Blanco CA. phenols and melanoidins as natural antioxidants in beer. structure, reactivity and antioxidant activity. Biomolecules. 2020;10(3):400. doi:10.3390/ biom 10030400
96. Snopek L, Mlcek J, Sochorova L, et al. Contribution of red wine consumption to human health protection. Molecules. 2018;23 (7):1684. doi:10.3390/molecules23071684

97. Kessing BF, Bredenoord AJ, Saleh CM, Smout AJ. Effects of anxiety and depression in patients with gastroesophageal reflux disease. Clin Gastroenterol Hepatol. 2015;13(6):1089-1095. doi:10.1016/j.cgh.2014.11.034

98. Fujiwara Y, Arakawa T, Fass R. Gastroesophageal reflux disease and sleep. Gastroenterol Clin North Am. 2013;42(1):57-70. doi:10.1016/j.gtc.2012.11.011

99. Shepherd K, Ockelford J, Ganasan V, Holloway R, Hillman D, Eastwood P. Temporal Relationship Between Night-Time Gastroesophageal Reflux Events and Arousals From Sleep. Am J Gastroenterol. 2020;115(5):697-705. doi:10.14309/ ajg.0000000000000627

100. Herregods TV, van Hoeij FB, Oors JM, Bredenoord AJ, Smout AJ. Effect of Running on Gastroesophageal Reflux and Reflux Mechanisms. Am J Gastroenterol. 2016;111(7):940-946. doi:10.1038/ajg.2016.122

101. de Mello DGL, Herbella F, Katayama RC. Transdiaphragmatic Pressure Gradient (TPG) Has a Central Role in the Pathophysiology of Gastroesophageal Reflux Disease (GERD) in the Obese and it Correlates with Abdominal Circumference but Not with Body Mass Index (BMI). Obes Surg. 2020;30 (4):1424-1428. doi:10.1007/s11695-019-04345-x

102. Mawditt C, Sasayama K, Katanoda K, Gilmour S. The clustering of health-related behaviours in the adult Japanese population. J Epidemiol. 2020. doi:10.2188/jea.JE20200120

103. Allen L, Williams J, Townsend N, et al. Socioeconomic status and non-communicable disease behavioural risk factors in low-income and lower-middle-income countries: a systematic review. Lancet Glob Health. 2017;5(3):e277-e289. doi:10.1016/ S2214-109X(17)30058-X

104. Hiza HA, Casavale KO, Guenther PM, Davis CA. Diet quality of Americans differs by age, sex, race/ ethnicity, income, and education level. J Acad Nutr Diet. 2013;113(2):297-306. doi:10.1016/j. jand.2012.08.011
Therapeutics and Clinical Risk Management

\section{Publish your work in this journal}

Therapeutics and Clinical Risk Management is an international, peerreviewed journal of clinical therapeutics and risk management, focusing on concise rapid reporting of clinical studies in all therapeutic areas, outcomes, safety, and programs for the effective, safe, and sustained use of medicines. This journal is indexed on PubMed Central, CAS,

\section{Dovepress}

EMBase, Scopus and the Elsevier Bibliographic databases. The manuscript management system is completely online and includes a very quick and fair peer-review system, which is all easy to use. Visit http://www.dovepress.com/testimonials.php to read real quotes from published authors. 